University of Texas at El Paso

\title{
DigitalCommons@UTEP
}

Open Access Theses \& Dissertations

2017-01-01

\section{Optimization of Neural Network Architecture for Classification of Radar Jamming FM Signals}

Alberto Soto

University of Texas at El Paso, asoto21@miners.utep.edu

Follow this and additional works at: https://digitalcommons.utep.edu/open_etd

Part of the Artificial Intelligence and Robotics Commons, and the Electrical and Electronics Commons

\section{Recommended Citation}

Soto, Alberto, "Optimization of Neural Network Architecture for Classification of Radar Jamming FM Signals" (2017). Open Access Theses \& Dissertations. 557.

https://digitalcommons.utep.edu/open_etd/557

This is brought to you for free and open access by DigitalCommons@UTEP. It has been accepted for inclusion in Open Access Theses \& Dissertations by an authorized administrator of DigitalCommons@UTEP. For more information, please contact lweber@utep.edu. 


\title{
OPTIMIZATION OF NEURAL NETWORK ARCHITECTURE FOR CLASSIFICATION OF RADAR JAMMING FM SIGNALS
}

\author{
ALBERTO SOTO \\ Master's Program in Electrical Engineering
}

APPROVED:

Benjamin C. Flores, Ph.D., Chair

Sarkodie-Gyan Thompson, Ph.D.

Patrick S. Debroux, Ph.D.

Melvin Robinson, Ph.D.

Charles Ambler, Ph.D.

Dean of the Graduate School 
Copyright (C)

by

Alberto Soto

2017 


\section{Dedication}

To my parents Alberto and Norelia Soto, whose unconditional love and support made me the person that I am today. Gracias por todo. Los quiero. 


\title{
OPTIMIZATION OF NEURAL NETWORK ARCHITECTURE FOR CLASSIFICATION OF RADAR JAMMING FM SIGNALS
}

\author{
by \\ ALBERTO SOTO, BSEE
}

\begin{abstract}
THESIS
Presented to the Faculty of the Graduate School of The University of Texas at El Paso

in Partial Fulfillment

of the Requirements

for the Degree of
\end{abstract}

MASTER OF SCIENCE

DEPARTMENT OF ELECTRICAL AND COMPUTER ENGINEERING

THE UNIVERSITY OF TEXAS AT EL PASO

May 2017 


\section{Acknowledgements}

First and foremost, I have to thank my family for all their support and encouragement. Especially my parents who have dedicated their lives to provide me with the tools and inspiration needed to advance in life. Special thanks to my siblings for always being there for me when hard times knocked at my door. My brother Saul is a very intelligent individual who also happens to be a great teacher. Through him I learned many things. My sister Luz, with her strong character and success, fuels my ambition to better myself and to strive for greatness. My best friend and twin sister Laura, with her strong determination and strong work ethic, she taught me to work hard for the things that I aspire to achieve. I also thank her for always being by my side.

I would like to sincerely thank my advisor, Dr. Benjamin C. Flores, for allowing me to become a researcher under his guidance. His ongoing support motivated me to aspire for a Master of Science degree and taught me that with hard work anything is possible. Dr. Flores is a great problem solver and extremely knowledgeable, qualities that immensely helped in the advancement of this study. I am extremely thankful for all the opportunities he has afforded me and for giving me a home in his radar lab. I would also like to express my gratitude towards Dr. Patrick S. Debroux and to Dr. Berenice Verdin for their invaluable contributions to this work. Their willingness to provide me with support and assistance made my journey of research an enjoyable experience. My thesis committee members deserve my wholehearted thanks as well. I am indebted to Dr. Patricia A. Nava for taking time from her busy schedule to help me improve my work. Her knowledge of neural networks became a vital asset for the formulation of this thesis. 
Last but not least, I want to give thanks to all my friends for never leaving my side as I took on this journey. It is through you all that I found assurance during tough times. My desire to make you all proud enabled me to keep working hard and to always reach for my goals. 


\begin{abstract}
Radar jamming signal classification is valuable when situational awareness of radar systems is sought out for timely deployment of electronic support measures. Our thesis shows that artificial neural networks can be utilized for effective and efficient signal classification. The goal is to optimize an artificial Neural Network (NN) approach capable of distinguishing between two common radar waveforms, namely bandlimited white Gaussian jamming noise (BWGN) and the ubiquitous linearly frequency modulated (LFM) signal. This is made possible by creating a theoretical framework for $\mathrm{NN}$ architecture testing that leads to a high probability of detection (PD) and a low probability of false alarm (PFA). It is assumed that some signal processing is required to improve the odds of correct signal classification. Therefore, our approach is to train the NN with a matrix populated with $\mathrm{M}$ spectra, where each spectrum is a sequence of $\mathrm{N}$ samples. Following standard procedure, $70 \%$ of the spectra are used for training, $15 \%$ for validation, and the remaining $15 \%$ for testing. A multilayered feedforward network topology is chosen for neuron arrangement and interconnection. Extensive experimentation is required to reveal the optimal number of hidden neurons, the optimal number of hidden layers, and lastly, the most efficient learning algorithm. Results show that a 98.5\% PD and a 1.7\% PFA of classifying between signal and noise are achieved when utilizing 10 or more hidden neurons. Changing the number of hidden layers has no significant effect in performance but the use of one hidden layer does reduce training time. Furthermore, the effect of using the Scaled Conjugate Gradient learning algorithm nearly yields a $100 \%$ PD. An architecture with at least ten hidden neurons, one hidden layer, and a Scaled Conjugate Gradient for training algorithm encapsulates an optimal architecture for our application.
\end{abstract}




\section{Table of Contents}

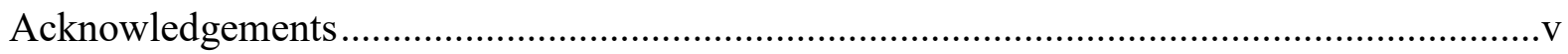

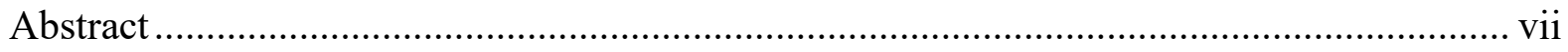

Table of Contents ................................................................................................... viii

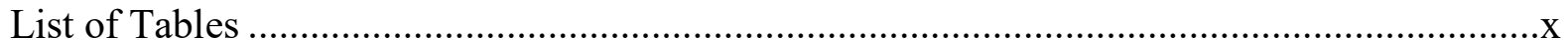

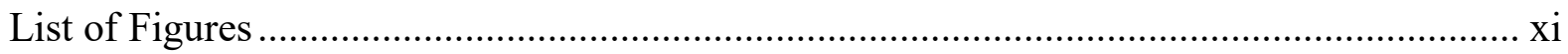

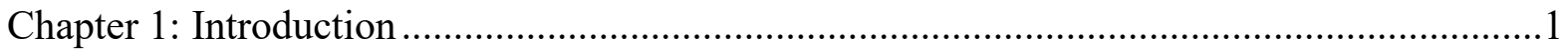

1.1 Motivation and Problem Statement ................................................................... 1

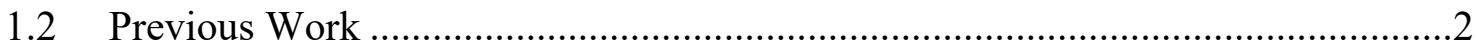

1.3 Methodology, Proposed Solution and Goal .........................................................

Chapter 2: Artificial Neural Network ...........................................................................5

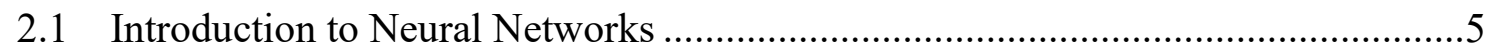

2.2 Applications of Neural Networks .............................................................6

2.3 Basic Structure of the Artificial Neuron ...........................................................

2.4 Multilayer Feedforward Network ............................................................

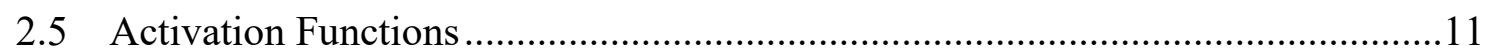

2.6 Supervised and Unsupervised Learning .................................................. 14

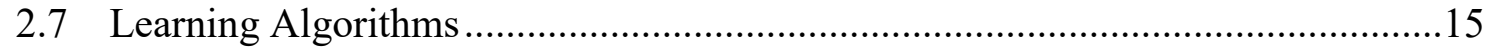

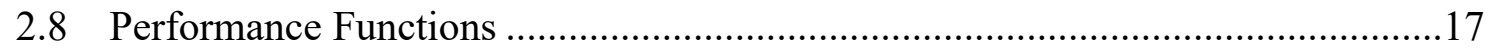

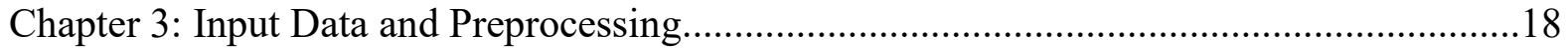

3.1 Linear Frequency Modulated (LFM) Signal ................................................ 18

3.2 Bandlimited White Gaussian Noise (BWGN) .................................................19

3.3 Generation of Discrete Linear FM Signals ...................................................20

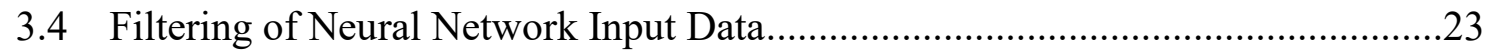

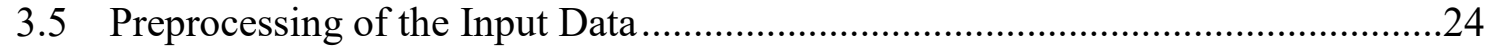

3.6 Organization of Input Data into Vector Form.................................................27

Chapter 4: Analysis of Multiple Neural Network Architectures and Learning Algorithms ....30

4.1 Neural Network Architecture with Varying Number of Hidden Neurons.............33

4.2 Neural Network Architecture with Varying Number of Hidden Layers ...............37

4.3 Assessment of Different Learning Algorithms for a Multilayer Network.............38 
Chapter 5: Conclusions

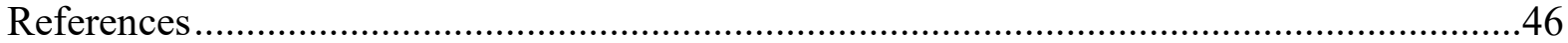

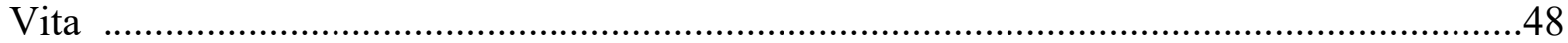




\section{List of Tables}

Table 4.1: Neural network performance with varying number of hidden neurons. 34

Table 4.2: Neural network performance for the Resilient Backpropagation learning algorithm with varying number of hidden neurons. 36 Table 4.3: Neural network performance for the Conjugate Gradient with Powell/Beale Restarts learning algorithm with varying number of hidden neurons. 36 Table 4.4: Neural network performance for the Polak-Ribiére Conjugate Gradient learning

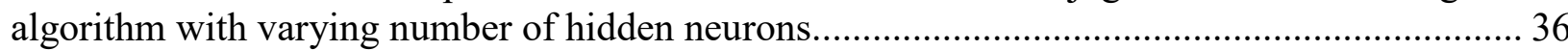
Table 4.5: Neural network performance for the Variable Learning Rate Backpropagation learning algorithm with varying number of hidden neurons................................................................ 37

Table 4.6: Standard deviations of the performances for the plot in Figure 4.5 ......................... 38

Table 4.7: Training parameters for the Resilient Backpropagation (RP) learning algorithm....... 39

Table 4.8: Training parameters for the Scaled Conjugate Gradient (SCG) learning algorithm... 39 Table 4.9: Training parameters for the Conjugate Gradient with Powell/Beale Restarts (CGB) learning algorithm.

Table 4.10: Training parameters for the Variable Learning Rate Backpropagation (GDX) learning algorithm. 40

Table 4.11: Training parameters for the Polak-Ribiére Conjugate Gradient (CGP) learning algorithm

Table 4.12: Standard deviations of the performances for the graph in Figure 4.6. 42 


\section{List of Figures}

Figure 1.1: Diagram denoting the process used to utilize a NN for signal classification............. 4

Figure 2.1: A simple artificial neuron............................................................................ 8

Figure 2.2: Feedforward multilayer network. ............................................................. 10

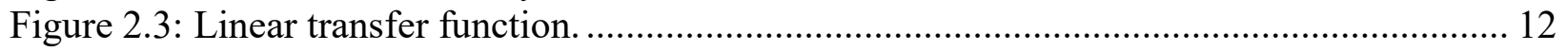

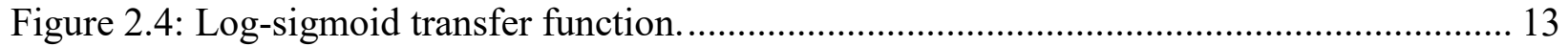

Figure 2.5: Hyperbolic tangent sigmoid transfer function.................................................. 13

Figure 3.1: LFM signal representation in the time domain. ................................................. 22

Figure 3.2: BWGN representation in the time domain.................................................. 22

Figure 3.3: Magnitude response of a bandpass Chebyshev Type 1 filter. .................................. 24

Figure 3.4: Power spectrum of a LFM signal with $\mathrm{SNR}=30 \mathrm{~dB}, \beta_{\mathrm{n}}=0.2$ and $\mathrm{f}_{\mathrm{n}}=0.2 \ldots \ldots \ldots \ldots \ldots .25$

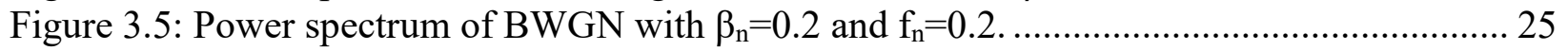

Figure 3.6: Power spectrum of a LFM signal with $\mathrm{SNR}=5 \mathrm{~dB}, \beta_{\mathrm{n}}=0.15$, and $\mathrm{f}_{\mathrm{n}}=0.05 \ldots \ldots \ldots \ldots .26$

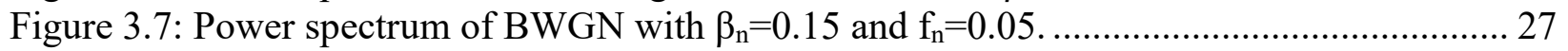

Figure 3.8: Organization of power spectra into a NxM input matrix. ...................................... 28

Figure 3.9: Target matrix containing the classification codes used to distinguish between LFM

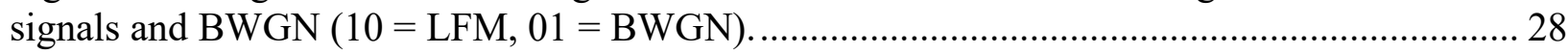

Figure 4.1: Neural network diagram with a focus on hidden neurons.................................. 30

Figure 4.2: Neural network diagram with a focus on hidden layers. ....................................... 30

Figure 4.3: Sample confusion matrix for a neural network architecture of 512-10-2 ............... 33

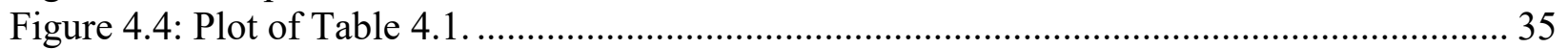

Figure 4.5: Neural network performance with varying number of hidden layers. ..................... 38

Figure 4.6: Assessment of neural network performance with different learning algorithms. ...... 41 


\section{Chapter 1: Introduction}

The human brain is a biological network that is comprised of about 100 billion nerve cells called neurons. Each neuron communicates with many others, together giving rise to the overwhelming richness and complexity of the brain's neural circuit [1]. The immense interconnectivity of neurons, along with the capability to process information in parallel is what gives the brain the ability to learn, and among many other functions, recognize intricate patterns. This incredible ability to learn inspired the development of artificial neural networks, which attempt to mimic the biological functionality of the human brain. An artificial Neural Network $(\mathrm{NN})$ is a computational system that loosely models the neuronal structure of the mammalian cerebral cortex. The list of NN applications, the money invested in NN software and hardware, and the depth of interest in these devices have been growing rapidly to solve complex problems [2] encountered in defense applications. Having said that, this project is an outgrowth of a study funded by the Army Research Laboratory.

\subsection{Motivation and Problem Statement}

Radar jamming signal detection and classification against a background of interference is considered a general radar problem. For military purposes the 'general radar problem' includes searching, interception, localization, analysis and identification of radiated electromagnetic energy which is commonly known as radar electronic support measures [3]. Motivation for this research arises from aiding the movement of countermeasures against unfriendly radar jamming signals by narrowing our focus to classifying their radiated electromagnetic energy.

For the timely deployment of countermeasures, attaining the information mentioned above can prove crucial for the purpose of situational awareness of radar systems. In order to realize our 
purpose, we propose to develop a systematic approach to constructing an optimal NN capable of classifying Linear Frequency Modulated (LFM) signals from Bandlimited White Gaussian Noise (BWGN). The distinctive task of classification by these networks can be expected to yield desirable results given that NNs are designed to extract existing patterns from noisy data [4].

\subsection{Previous Work}

Neural networks and their ability to perform radar signal classifications has been the catalyst for extensive investigation [3, 5-9]. This has led to an increasing effort in the research to optimize $\mathrm{NN}$ architectures and topologies to ensure acceptable and high efficiency classification results $[10,11]$. Only by trial and error can a user, experimentally, find the optimal NN architecture suited for their applications $[6,7]$. Although not related to signal classification other work also corroborates the previous statement $[4,12]$.

Petrov, Jordanov, and Roe [3] attempted to increase classification accuracies between civilian and military signals by examining two $\mathrm{NN}$ architectures with different neural topologies in the output layer: 1 output neuron in the first case and 2 output neurons in the second. The rest of the architectural characteristics were kept identical. The first architecture used 1 binary output neuron to code the outputs as 0 (civilian signal) and 1 (military signal). The latter used 2 binary output neurons to code the outputs as 10 (civilian signal) and 01 (military signal). By doing so they were able to study the effects of changing the output architecture of the network while only having two classes to choose from. Classifier accuracies for the testing sets in the second case were slightly greater, concluding that having a neuron for each class can promote better results.

In their search for a radar signal classifier, Ahalt et al. [8] proposed no method for finding an optimal NN architecture but they did examine five cases containing one hidden layer with 5, 10, 20, 30, and 40 hidden neurons in each instance. In addition, they inspected two more cases: 
one case with 2 hidden layers and the other with 3 hidden layers, whereas the number of hidden neurons was kept fixed at 10 within each hidden layer. Like Hara et al. [13], Ahalt and collaborators concluded that NN performance is insensitive to changes in network architecture. Nonetheless, different learning algorithms were not considered nor additional network architectures explored.

\subsection{Methodology, Proposed Solution And Goal}

In this thesis, we are interested in finding a theoretical framework to determine an optimal NN architecture for which high classification accuracies are achieved, even when the Signal-toNoise Ratio (SNR) is low. All the NNs, as well as the generated LFM signals and BWGN, were simulated using MATLAB®. The analysis begins by generating a database with an array of different LFM signals and BWGN which will later be processed to obtain their spectra. Given that preliminary results used to formulate our thesis suggested that the moments of the spectrum characterize the signal quite efficiently, we opt to populate a matrix containing $M$ spectra. The matrix is fed to the $\mathrm{NN}$ which uses $70 \%$ of the spectra for training, $15 \%$ for validation, and $15 \%$ for testing.

To analyze the different architectures, we propose a similar approach to work done by Ahalt et al. [8] to study the varying number of hidden neurons and hidden layers. In addition, a closer look is given to the different training algorithms for pattern recognition applications. The algorithms explored in this work are: Resilient Backpropagation (RP), Scaled Conjugate Gradient (SCG), Conjugate Gradient with Powell/Beale Restarts (CGB), Polak-Ribiére Conjugate Gradient (CGP), and Variable Learning Rate Backpropagation (GDX). We experimentally determine the number of hidden neurons, then the number of hidden layers, and finally the most efficient training algorithm. 
The goal of this project is to develop an optimal network capable of classifying radar jamming signals with a Probability of Detection (PD) of 95\% or higher and a Probability of False Alarm (PFA) of $1.5 \%$ or lower at a threshold SNR value of 5dB. The diagram in Figure 1.1 summarizes the methodology developed to carry out this research, commencing with the signal inputs and ending with the resulting $\mathrm{NN}$ classification results.

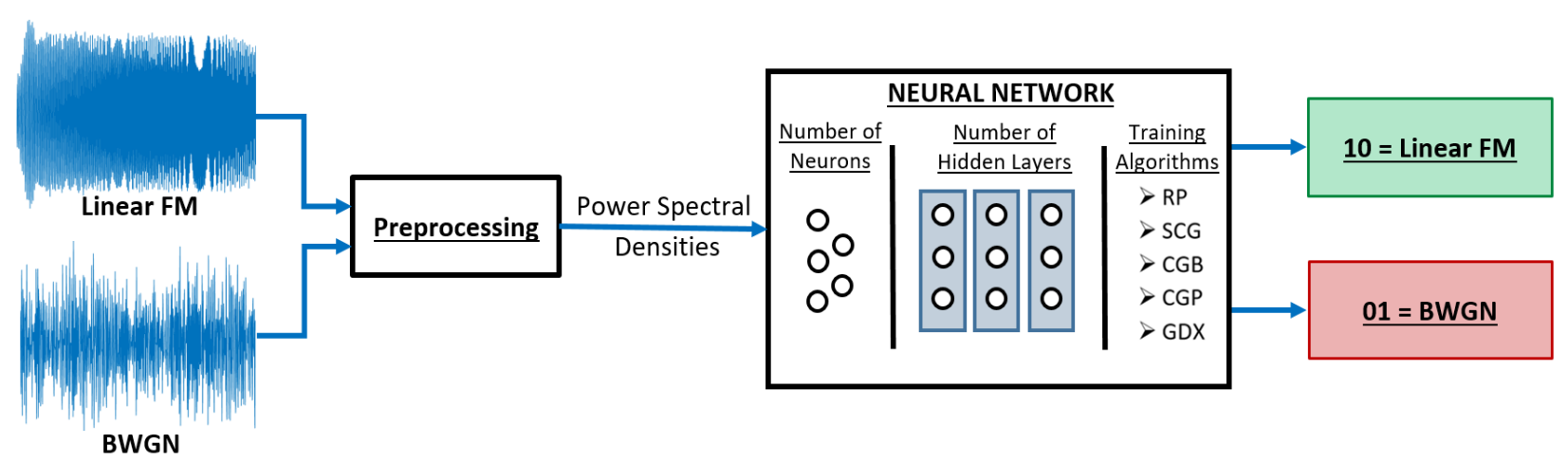

Figure 1.1: Diagram denoting the process used to utilize a NN for signal classification.

This thesis has the following structure: Chapter 2 contains a review on NNs, namely an introduction, applications, basic neuron model, architectures and learning algorithms. Chapter 3 addresses the simulation of radar jamming FM signals and BWGN as well as the preprocessing of the data, meaning the filtering and the computation of spectra. In addition, the organization of the resulting data into a $\mathrm{NN}$ input matrix is discussed in the same chapter. Chapter 4 is dedicated to discussing the PD and PFA levels obtained for various NN architectures for a SNR level of 5dB. Finally, chapter 5 summarizes the major findings derived from this work. 


\section{Chapter 2: Artificial Neural Network}

\subsection{INTRODUCTION TO NEURAL NETWORKS}

Artificial NNs are an attractive alternative to single processor computers and can be seen as an important and widely applicable constituent in future computational technologies. From here on, artificial neural network and the designation $\mathrm{NN}$ will be used interchangeably. Rather than having single processors, NNs rely on dense arrangements of interconnections and simple processors which give rise to their immense capability of parallel data processing. In a NN each processor is linked to many of its neighbors, so that there are many more interconnects than processors [14]. In all reality, the number of interconnections is far greater than the number of processing units. As stated by Judith E. Dayhoff, the power of the NN lies in the tremendous number of interconnections.

While a CPU has the characteristic task of processing hundreds or thousands of basic commands in a clock cycle, a NN's single processing unit performs from one to a few number calculations. The difference is patently observable when contrasting the methods concerning command/calculation executions: a traditional single processor computer executes commands successively whereas the NN activates all of its processing units simultaneously despite the fact that each unit calculates only a few calculations. Moreover, traditional memory schemes where particular pieces of information are stored in particular locations in memory, contrast with NN architectures where information is encoded in a distributed fashion. This ensues because information is shared by many of the NN's processing units.

Furthermore, unlike CPUs, a NN system can undergo partial destruction of the network and may still be able to function correctly [14]. Due to its distributed storage schemes, the manner in which NNs represent information can be redundant. Even though this characteristic can be built 
into other types of systems, it is mentioned by Dayhoff that NNs have a natural way to organize and implement this redundancy, resulting in a naturally fault- or error-tolerant system. But what has triggered the most interest in NNs is that these models, which are similar to biological nervous systems, can actually be made to do useful computations, and, furthermore, the capabilities of the resulting systems provide an effective approach to previously unsolved problems [14].

\subsection{Applications OF NeUral Networks}

In areas where intractable solutions of traditional computing have fallen short, artificial NNs have appeared to lend an appropriate approach to solving some of today's problems. These networks have been used in solving problems that require signal filtering, pattern recognition, pattern mapping, dealing with noisy data, pattern completion, image processing and systems that learn or adapt during use. With so many applications, the study of NNs has developed as an interdisciplinary field. Medicine, banking and defense are some of the fields attracted to the individuality of NNs.

The main focus revolving around this thesis will be in the field of defense. Radar electronic support measures are highly sought out by the military for radar system awareness. Environments are increasing in density where electromagnetic energy and radar jamming signals are becoming more difficult to detect and classify. Traditional algorithms for signal recognition and analysis are

highly complex, computationally intensive, often rely on heuristics, and require humans to verify and validate the analysis [7]. Artificial NNs will be used as an alternative approach that hopes to increase the success of radar electronic support measures. 


\subsection{Basic Structure of the Artificial Neuron}

As mentioned earlier, NNs are made up of processing units and interconnections. Processing units are commonly known as neurons, units, cells, or nodes. In this work we will adopt the title of neurons when addressing a processing unit. Neurons have an activation state that behaves as a function to its inputs. When activated, a neuron uses an activation function to output a signal. A single neuron is directly connected to other neurons and each connection has an associated weight that multiplies the signals transmitted between these processing units. Weights are the information that is adjusted by the $\mathrm{NN}$ to solve problems [15], which supports the notion that the power of NNs lies within its interconnections.

It is a common misconception to think of NNs as a black box considering that explanations depicting their behavior are often obscured. Nevertheless, the computations performed in the network are mathematical, and typically are similar to other mathematical methods already in use. Although, large NN systems may sometimes act in surprising ways, their internal mechanisms are neither mysterious nor incomprehensible [14]. The feat to mathematically examine large NNs is both time consuming and impractical but, regardless, the analysis can be done. The characteristics of these networks can be explained through the mathematical structure of a simple NN model.

A typical processing unit found in artificial NNs is illustrated in Figure 2.1. Neuron $Y$ receives inputs from $X_{1}, X_{2}, X_{n}$ and a component $X_{0}$ (usually has a set activation value of 1.0) used to implement a bias weight. The weights on the connections from the inputs $X_{0}, X_{1}, X_{2}$ and $X_{n}$ to neuron $\mathrm{Y}$ are $w_{0}$ (bias), $w_{1}, w_{2}$ and $w_{n}$, respectively. The mathematical model of the neuron computing a weighted sum of all the inputs, $y_{-}$in, is described as follows:

$$
y_{-} \text {in }=\sum_{i=0}^{n} w_{i} x_{i}
$$


where $x_{i}$ is the input signals traveling from $X_{i}$ to neuron $\mathrm{Y}$ and $w_{i}$ is the corresponding weights of those interconnections. In order for the neuron to fire its signal, either as an output or to another neuron, it has to become activated. The activation $y$ of neuron $\mathrm{Y}$ is given by

$$
y=f\left(y_{-} i n\right)
$$

where $f()$ is the activation function and whose argument is $y_{-} i n$.

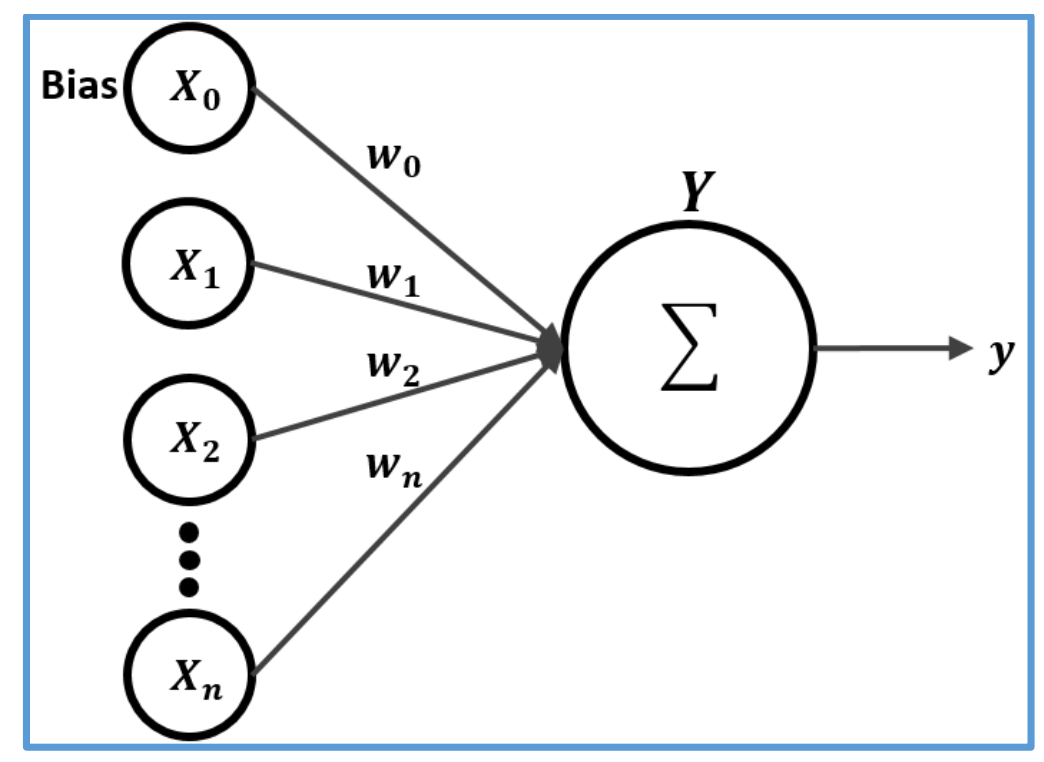

Figure 2.1: A simple artificial neuron.

\subsection{Multilayer FeEdForward Network}

Artificial NNs are made up of many neurons and it is convenient to visualize them arranged in layers. The arrangement of neurons into layers and the connection patterns within and between layers is called the net architecture [15]. It is typical for neurons in the same layer to behave in the same manner: i.e., neurons will share characteristics like their activation function and pattern of interconnectivity to other neurons. The net architecture allows for the classification of NNs to be 
categorized either as a single or multilayer networks. Both networks are examples of feedforward networks - networks in which the signals flow from the input units to the output units, in a forward direction [15].

As the name implies, single layer networks have only one layer where the inputs are fully connected to the output neurons. An example of a single layer architecture is previously shown in Figure 2.1 with the representation of a single neuron network. On the other hand, a multilayer network is a NN with one or more layers of neurons. An example of a multilayer network is shown in Figure 2.2.

A multilayer $\mathrm{NN}$ is composed of one input layer, one output layer and a customizable number of hidden layers. The hidden layer is neither an input nor an output and is not seen by the user and thus, consequently, obtained the name hidden layer. The input layer serves as a port for an input pattern (represented as a vector) that will be fed to the network and characteristically equals in size to the input pattern vector. The output layer contains as many neurons as there are classes. The number of hidden layers and the number of hidden neurons (neurons located within the hidden layer) are not fixed; it is important to mention that these parameters are dependent on the application at hand. Furthermore, hidden neurons are seen as "feature detectors" - units that respond to particular features that may appear in the input pattern [14]. 


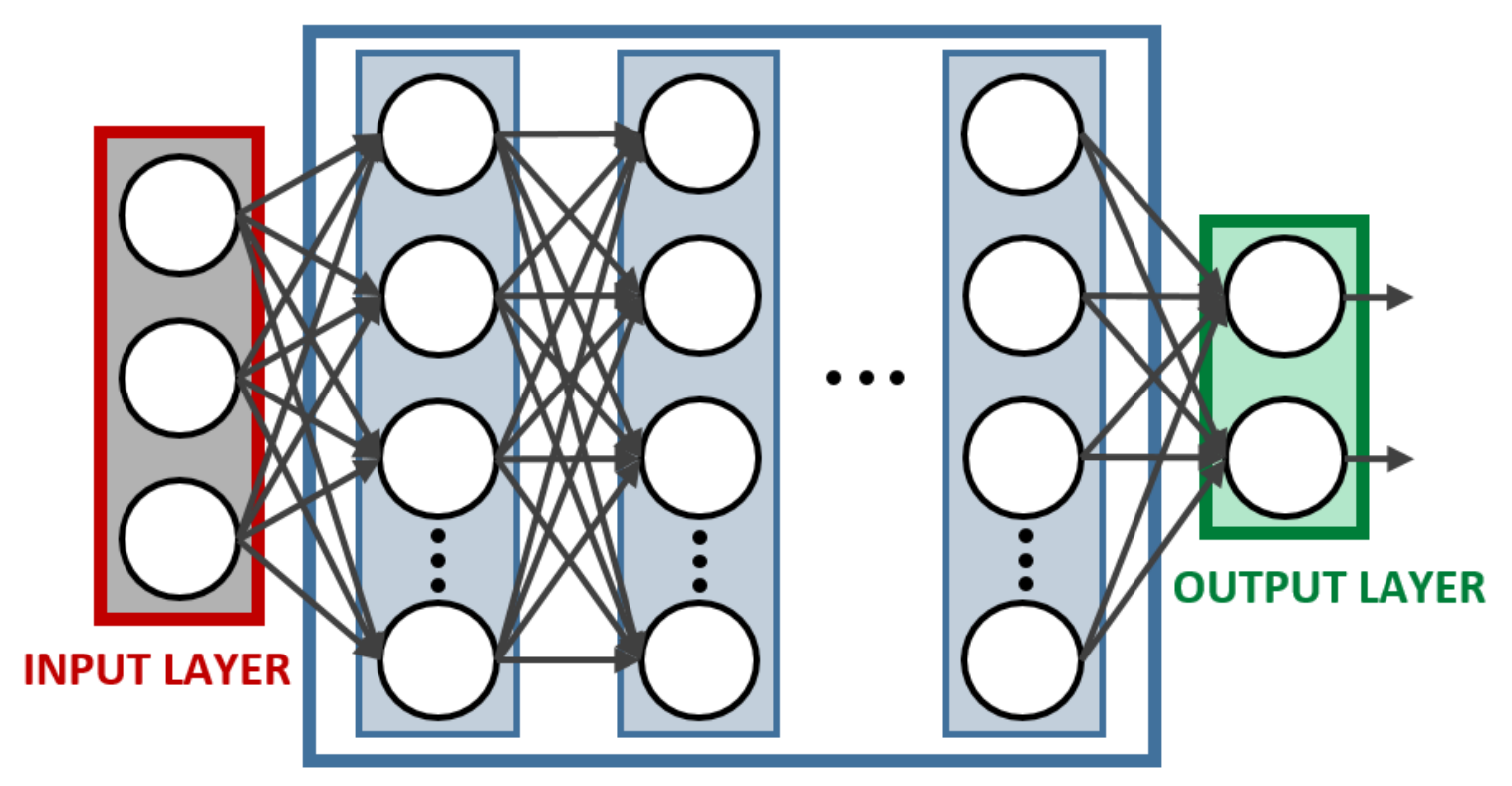

HIDDEN LAYERS

Figure 2.2: Feedforward multilayer network.

Problems like that of classification and pattern association are most commonly solved by networks with multilayer architectures. That being said, a multilayer network is the choice of topology for our application of radar jamming signal classification. Though the need for classification influences the choice in using a multilayer network, it does not uniquely determine its net architecture. In our simulations, the input layer equals the size of the input vector, two is the number of neurons in the output layer since there are only to classes being tested, and lastly, the number of hidden neurons and layers, as mentioned in $[5,6]$, will be determined experimentally by trial and error. In this work, finding an optimal architecture has brought about our interest in determining an efficient number of hidden layers and hidden neurons for our application. 


\subsection{ACtivation FunCtions}

As mentioned earlier, neurons utilize activation functions (also known as transfer functions) to take the sum of the inputs, whose signals are multiplied by their corresponding weight, to generate an output. Neurons can use any differentiable function (a function whose derivative exists at each point in its domain) to generate their output [16]. Training algorithms make use of the derivative of the activation function to calculate the optimal value of the weights. Three common activation functions used in multilayer networks are the linear transfer function, the log-sigmoid (logistic sigmoid) transfer function, and the hyperbolic tan-sigmoid (tangent sigmoid) transfer function.

Linear transfer functions, illustrated in Figure 2.3, are typically used with single layer networks. The mathematical model of the linear function is as follows:

$$
f(x)=x \quad \text { for all } x
$$

Linear output neurons are more appropriate for function fitting problems rather than pattern recognition applications [16]. Fausett states, that in order to achieve the advantages of multilayer nets, compared with the limited capabilities of single layer nets, nonlinear functions are required. Since the results of feeding a signal through two or more layers of a linear processing element, i.e. elements with linear activation functions, are no different from what can be obtained using a single layer [15]. For these reasons, the linear activation function is not employed in the NN simulations of this research.

The most popular activation functions are the sigmoid functions (S-shaped curves), which are also known as squashing functions. The log-sigmoid activation function, illustrated in Figure 2.4 , is a type of sigmoid function that squashes a neuron's output to be between 0 and 1 . The output of the log-sigmoid function is computed as follows: 


$$
f(x)=\frac{1}{1+e^{-x}}
$$

The tan-sigmoid function, illustrated in Figure 2.5, is also a sigmoid function, but in contrast to the log-sigmoid function, squashes a neuron's output to be between -1 and 1 . The output of the tan-sigmoid activation function is computed as follows:

$$
f(x)=\frac{2}{\left(1+e^{-2 x}\right)}-1
$$

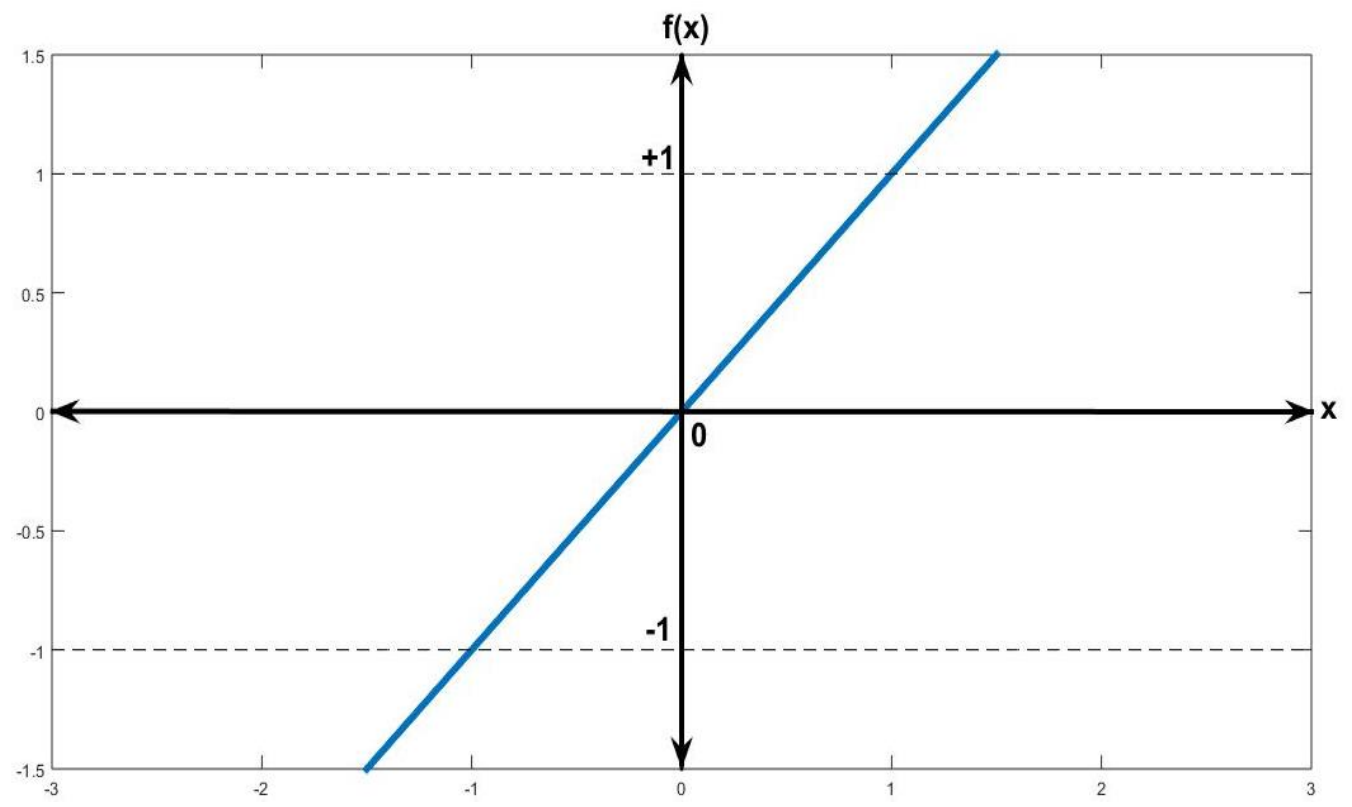

Figure 2.3: Linear transfer function. 


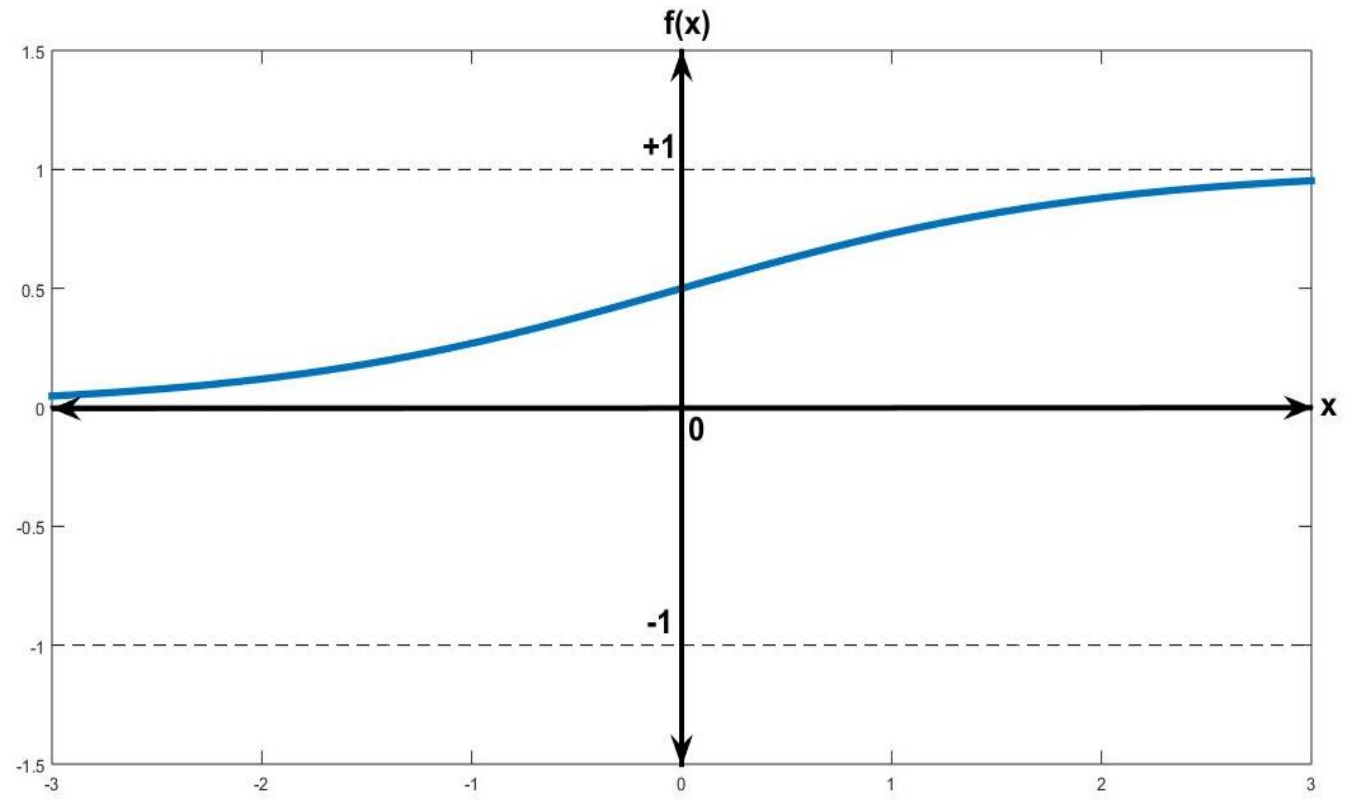

Figure 2.4: Log-sigmoid transfer function.

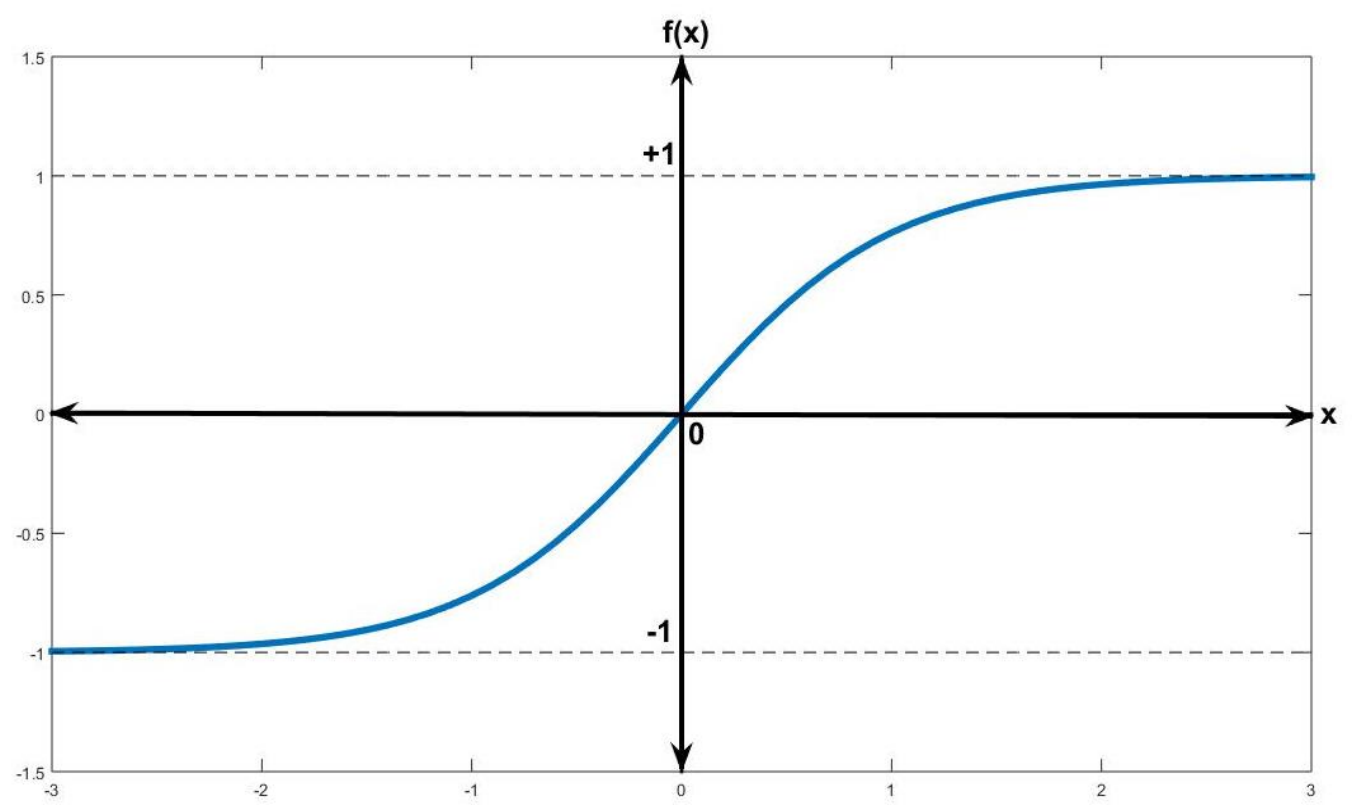

Figure 2.5: Hyperbolic tangent sigmoid transfer function. 
Sigmoid functions are especially advantageous for use in NNs trained with backpropagation, a type of training algorithm used in this research and discussed in subsection 2.7. Sigmoid functions have a simple relationship between the value of the function at a point and the value of the derivative at that point that reduces the computational burden during training [15]. The tan-sigmoid function is optimal for when training speed is important [17], meaning that it accelerates the convergence (adjustments of weights) of the backpropagation method. For this reason the tan-sigmoid function is our activation function of choice.

\subsection{SUPERVISED AND UNSUPERVISED LEARNING}

These systems are not programmed but rather learn by example. In order to tap into the functionality of NNs, the system must be presented with a training set consisting of a group of examples from which the network can learn [14]. The examples are presented in vector form and encompass the patterns that the user wishes for the NN to acquire. Data used in the input vector can be extracted from sensors, receivers, images and in our case, simulations. The training process can be categorized into two training scenarios: supervised and unsupervised.

Supervised learning is named in this manner because the NN is supplied with an input pattern along with a desired response. The desired response is often referred to as the target output and constitutes the correct answer, or correct classification for the given input pattern. If the actual output of the NN differs from the target output, an error is generated and then used to adjust the internal weights of the connections contained between neurons. If successful, the weights are adjusted to the point where the network will produce the correct answer for the pattern and minimize the error between the actual and the target data. Hence, the training is supervised. Classification and regression problems are commonly solved by employing supervised learning algorithms. 
Within unsupervised learning, the NN does not have the luxury of having a target answer, and thus adjusts its internal weights and arbitrarily self organizes the patterns into similar clusters. The goal of unsupervised learning is to model the underlying structure or distribution in the data in order to learn more about the input data. Unsupervised learning algorithms are usually associated with solving two kinds of problems: clustering and association. In clustering, the system is left to discover groupings in the data, e.g. grouping customers for similar purchasing behaviors. While in association the system discovers rules that describe large portions of your input data, e.g. people who buy new tires also tend to purchase a vehicle alignment service.

In summary, supervised learning algorithms train NNs to perform tasks such as identifying group membership or predicting a response, whereas unsupervised learning algorithms train NNs to generalize data that does not have a clear distinction and attempt to discover a structure within the input data. In our study, we strive for a system to perform the task of classification. Since LFM signals and BWGN are clearly distinct and their classes known, we are able to formulate a desired target to supplement the training of the system. Consequently, in this research, all the NN simulations utilize a supervised learning scenario for the training phase.

\subsection{LeARNing AlgorithmS}

The process of training a neural network involves tuning the values of the weights and biases of the network to optimize network performance [18]. In supervised learning there is a type of learning algorithm that is very popular when training multilayer networks, it is called backpropagation. Standard backpropagation is a gradient descent algorithm in which the network weights are moved along the negative of the gradient of the performance function [2].

Recall that multilayer feedforward networks have signals flowing from input units to output neurons in a forward direction. Alternately, the backpropagation algorithm is a learning rule 
in which weights and biases are adjusted by error-derivative vectors backpropagated through the network [2]. The error-derivative is calculated from the difference between the network's actual output and the desired output. After a multilayer network does a forward pass with the input patterns, the backpropagation training algorithm backpropagates the error-derivative with the aim of minimizing the error between the actual output and the desired output for the entire input training vectors [9].

As stated by Jordanov et al. [9], when training a multilayer network with the backpropagation technique, the learning/training period is generally a lengthy one, which is the principle weakness of the technique. Faster training algorithms have been developed to allow networks to converge from ten to one hundred times faster than the backpropagation algorithm. This study focuses on learning algorithms suited for pattern recognition problems. Since the process of computing the gradient and the Jacobian matrix by performing calculations backward through the network is applied in all the training algorithms listed below, the terminology backpropagation is not used in most of their names but it is still used for their derivation [18].

The five learning algorithms explored in this work are: Resilient Backpropagation (RP), Scaled Conjugate Gradient (SCG), Conjugate Gradient with Powell/Beale Restarts (CGB), PolakRibiére Conjugate Gradient (CGP), and Variable Learning Rate Backpropagation (GDX). Detailed summaries of each algorithm exceed the scope of this thesis. For detailed information about each training algorithm previously mentioned refer to the Neural Network Toolbox Manual [2] under the faster training section. Aside from training a network to successfully perform a classification, the time spent during the training phase can be an important deciding design factor when searching for an optimal learning algorithm. 


\subsection{Performance Functions}

When multilayer networks are learning, and using a variation of the backpropagation algorithm, a performance function is employed to determine the error between an actual output of the network and the desired output target. A common performance function that is usually used by the backpropagation algorithm is the Mean Squared normalized Error (MSE). It measures the network's performance according to the mean of squared errors [19]. The mathematical equation is as follows:

$$
M S E=\frac{1}{N} \sum_{i=0}^{N}\left(t_{i}-y_{i}\right)^{2}
$$

where $t_{i}$ is the target output, $y_{i}$ is the actual output and $\left(\frac{1}{N} \sum_{i=0}^{N}()^{2}\right)$ is the mean of the square of errors.

In addition, there is another function called the Cross-Entropy (CE) performance function which calculates a network's performance given its targets and outputs, just like the MSE function. The mathematical formula of the cross-entropy performance function of a pair of output-target elements is as follows:

$$
C E=-\sum_{i=1} t_{i} \log \left(y_{i}\right)
$$

where $t_{i}$ is the target output and $y_{i}$ is the network's actual output. To design a good classifier, the key is to minimizing the cross-entropy. Unlike the MSE, the cross-entropy function returns a result that heavily penalizes outputs that are extremely inaccurate, with very little penalty for fairly correct classifications [20]. Given this reason, the cross-entropy performance function is used for the simulations in this research. 


\section{Chapter 3: Input Data and Preprocessing}

The NN approach we propose uses signal information in the frequency domain to classify jamming signals. This chapter is dedicated to describing a technique developed for the purpose of generating a large number of testing signals. Furthermore, the signal processing required to prepare the data matrix used to test the NN network. Finally, we describe the organization of the data into vector form.

\subsection{Linear Frequency Modulated (LFM) SignaL}

The LFM signal, also known as a chirp signal, is most commonly used by radar systems to locate targets with high resolution. The signal has a frequency that varies linearly over a bandwidth, either increasing or decreasing, with time [21]. The mathematical expression of the LFM signal is

$$
s(t)=A e^{j 2 \pi\left(f_{0} t+\frac{k t^{2}}{2}\right)}+n(t) \quad 0 \leq t \leq T
$$

where $A$ is the amplitude of the signal, $f_{0}$ is the initial frequency, $k$ is the chirp rate of the waveform, and $n(t)$ is the injected noise. The bandwidth of the signal is given by

$$
\beta=k T
$$

where $T$ is the transmitted pulse duration that it takes to sweep between the initial frequency $f_{0}$ to the final frequency $f_{1}$. Equivalently, the bandwidth can be expressed as

$$
\beta=f_{1}-f_{0}
$$

The SNR in decibels is calculated as

$$
S N R_{d B}=10 \log _{10}\left(\frac{A^{2}}{\sigma^{2}}\right) .
$$


In terms of the SNR, the noise variance is

$$
\sigma^{2}=A^{2}\left(10^{-\left(\frac{S N R d B}{10}\right)}\right)
$$

which, in simulations, is used to compute the total additive noise $n(t)$, that corrupts the LFM signal to simulate real-world signals.

LFM signals are also used as effective and destructive jamming signals. A jamming system can be used to construct a replica of the transmitted LFM signal to create false targets [22]. Early detection of the jamming signal can reduce or even prevent unwanted interference to radar systems.

Salminen [23] advises that if the network is to operate in a noisy environment, then appropriate noise must be added to any synthetically generated training vector. In our case, the SNR of the LFM jamming signal is set to $5 \mathrm{~dB}$ to challenge the NN sufficiently while conducting an analysis of the $\mathrm{NN}$ architecture.

\subsection{BANDLIMITED White GaUSSIAN NOISE (BWGN)}

Another signal used for jamming purposes is the BWGN. To generate this random process, we start with zero mean White Gaussian Noise (WGN). The WGN sequence is complex and each sample is modeled as a Gaussian variable with a variance $\sigma^{2} \leq 1$. The noise is bandlimited so that it matches the power spectrum of the LFM signal. In order to limit its bandwidth, the signal is filtered. In the time domain this process is represented as

$$
n_{B L}(t)=h(t) * n(t)
$$

where

$$
h(t)=F^{-1}\{H(f)\}
$$

and $H(f)$ is a Chebyshev Type 1 bandpass filter to be further discussed in section 3.4. 


\subsection{GENERATION OF DisCRETE LiNEAR FM SignALS}

The training data set must include a variety of signal examples that prepare the NN for the generalization task [14]. Dayhoff emphasizes that it is with this variety of examples that a neural network can discover the distinguishing features needed to perform a classification task. Therefore, a method is needed to represent the needed information. In our application, we represent LFM signals and the random process of BWGN in terms of sample vectors which we simulate and use as input for the NN.

To create a database of signals and to facilitate the generation process we took equation (3.1) and represent it in terms of discrete samples as follows

$$
s(n)=A e^{\left(j 2 \pi f_{0}(n \Delta t)+j \pi k(n \Delta t)^{2}\right)} \quad 0 \leq n \leq N-1
$$

where $\mathrm{n} \Delta \mathrm{t}$ denotes discrete sampling. Now, the chirp rate is defined as

$$
k=\frac{\beta}{T}
$$

and the sampling interval is

$$
\Delta t=\frac{1}{f_{s}}
$$

where $f s$ is the sampling frequency. Therefore, the corresponding signal becomes

$$
s(n)=A e^{\left(j 2 \pi\left(\frac{f_{0}}{f_{S}}\right) n+j \pi n^{2}\left(\frac{\beta}{T f_{S}^{2}}\right)\right)} \quad 0 \leq n \leq N-1 .
$$

Furthermore, let the normalized initial frequency be

$$
f_{\text {norm }}=\frac{f_{0}}{f_{s}}
$$

and the normalized bandwidth 


$$
\beta_{\text {norm }}=\frac{\beta}{f_{s}}
$$

so that, by rearranging the terms in (3.11), the expression for the discrete LFM signal becomes

$$
s(n)=A e^{\left(j 2 \pi f_{n o r m}+j \pi \beta_{n o r m}\left(\frac{n^{2}}{(N-1)}\right)\right)} \quad n \leq n \leq N-1
$$

For testing purposes, we randomize the normalized initial frequency and bandwidth within their respective ranges

$$
0.02<f_{\text {norm }}<\left(1-\beta_{\text {norm }}\right),
$$

and

$$
0.05<\beta_{\text {norm }}<0.2
$$

where the limits are chosen to ensure the Nyquist Criterion. We can now generate a plethora of LFM signals with a varying number of initial frequencies and bandwidths while having total control of the number of samples per signal sequence. Figure 3.1 depicts a LFM signal with frequency ramping up as a function of time. A small amount of noise is added so the envelope is no longer constant. In contrast, Figure 3.2 shows a sequence of BWGN samples. In this case, the amplitude of the samples varies significantly and there is no discernible envelope. 


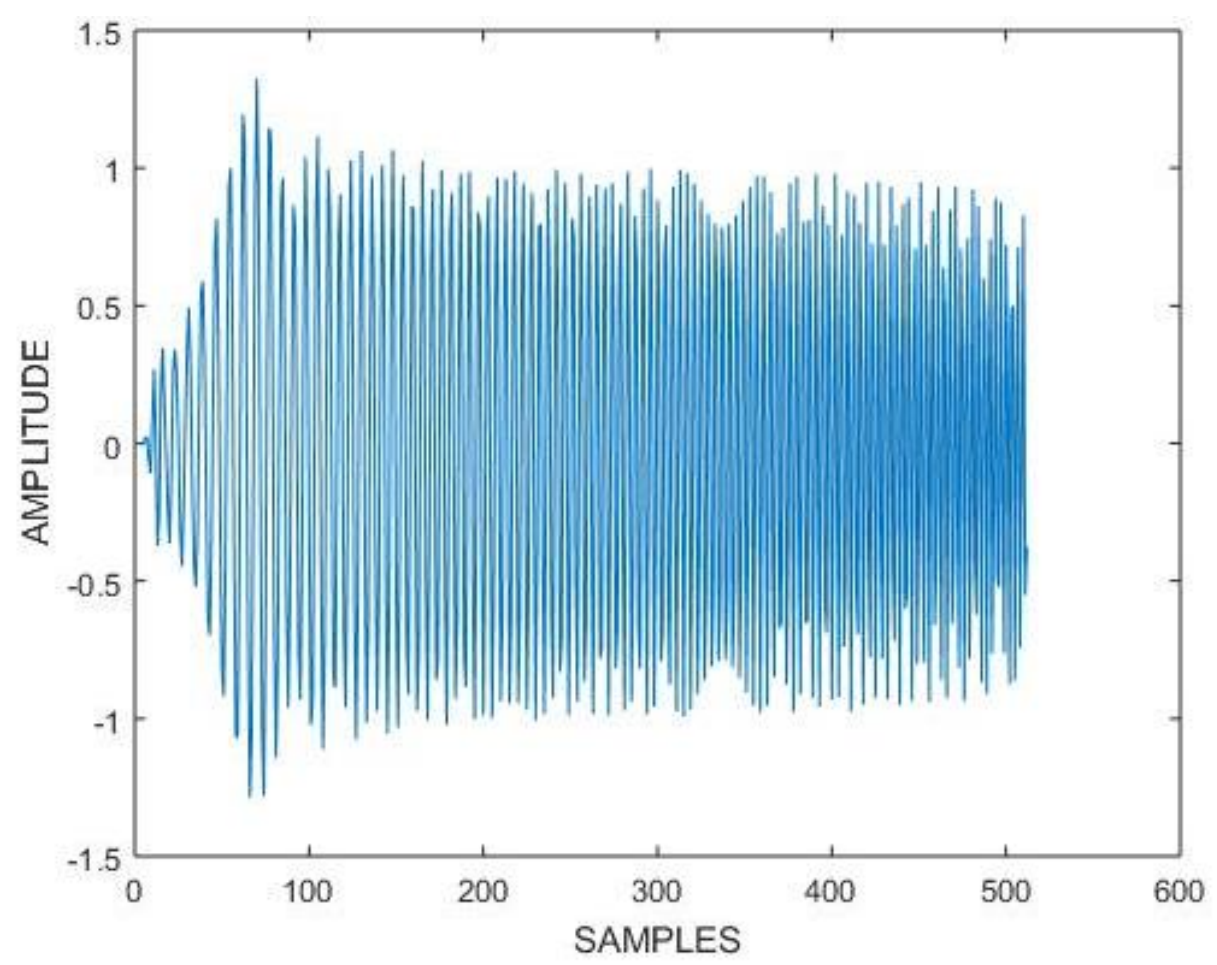

Figure 3.1: LFM signal representation in the time domain.

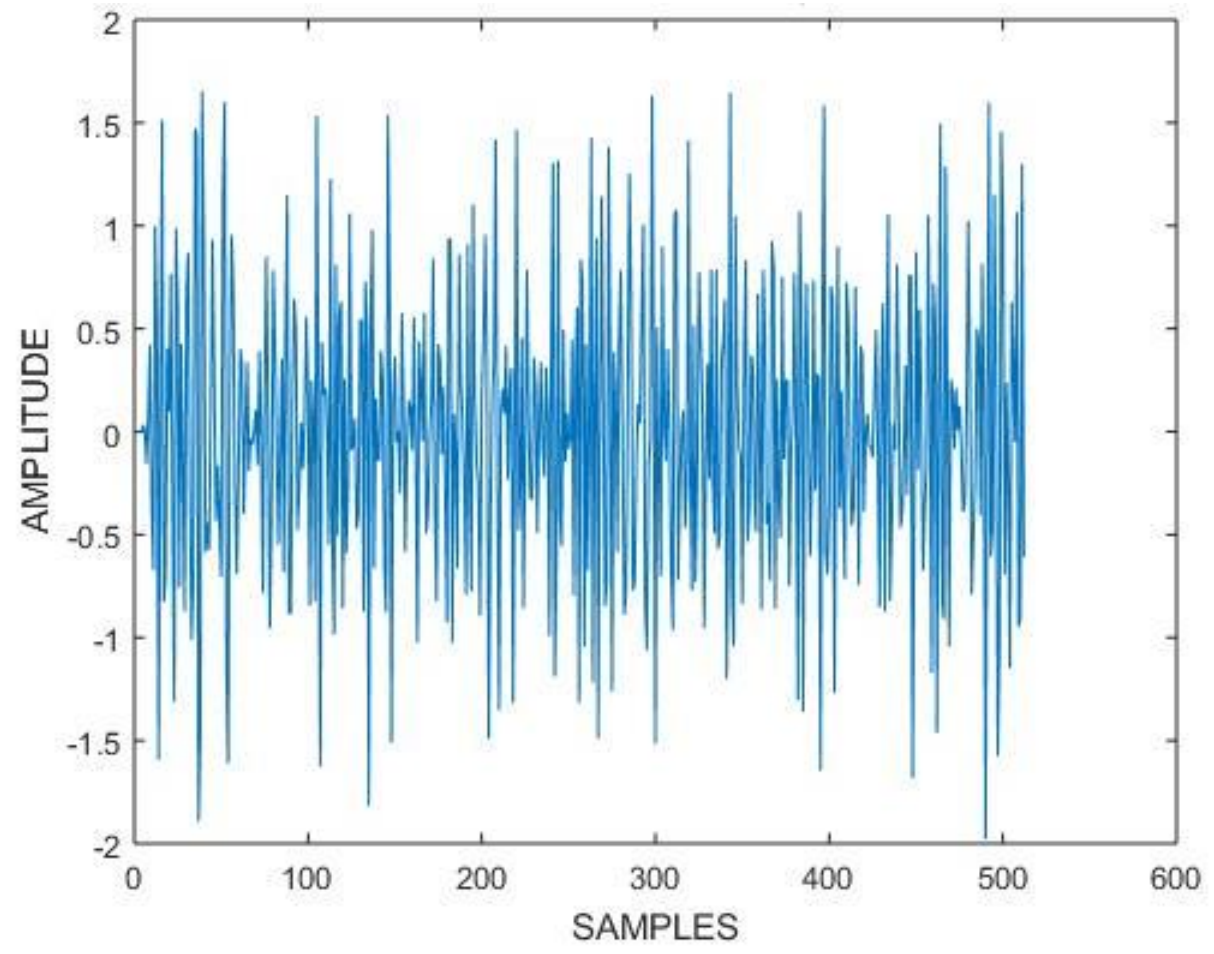

Figure 3.2: BWGN representation in the time domain. 
Dayhoff also states that the selection of the training data presented to the $\mathrm{NN}$ influences whether or not the network learns a particular task. The behavior and success of a NN are dependent on many variables. For instance, the type of architecture, the type of learning algorithm, and even the dataset used to train the network can all have positive and negative effects. Therefore, the generation of our data must be done with care by controlling the sampling rate of each signal in the input dataset to ensure they contain the same number of samples. In consequence, we can avoid a difference in the number of samples from affecting the performance of the NN.

\subsection{Filtering OF NeURAL NetWork INPUT DATA}

Modern radar systems utilize a wide instantaneous bandwidth in order to detect a variety of different targets. As a result these systems are susceptible to noise power spread over a wide spectrum [23]. A common practice is to include a filter in the radar receiver that aligns with the bandwidth of the received signal to limit noise and thus increase the SNR.

We choose a bandpass Chebyshev Type 1 filter to remove unwanted noise outside of the bandwidth of the testing signal. The passband corner frequencies of the filter matches the bandwidth of the signal and offers a steep roll-off as shown in Figure 3.3. The peak-to-peak passband ripple and the stopband attenuation are assigned values of $0.1 \mathrm{~dB}$ and $40 \mathrm{~dB}$, respectively. The function cheblord in MATLAB calculates the minimum order needed to meet a set of given filter design specifications for a specified LFM signal. In addition, for the purpose of simulating BWGN, we filter the Gaussian noise with the bandpass Chebyshev Type 1 filter to produce bandwidths similar to those of a LFM signal. 


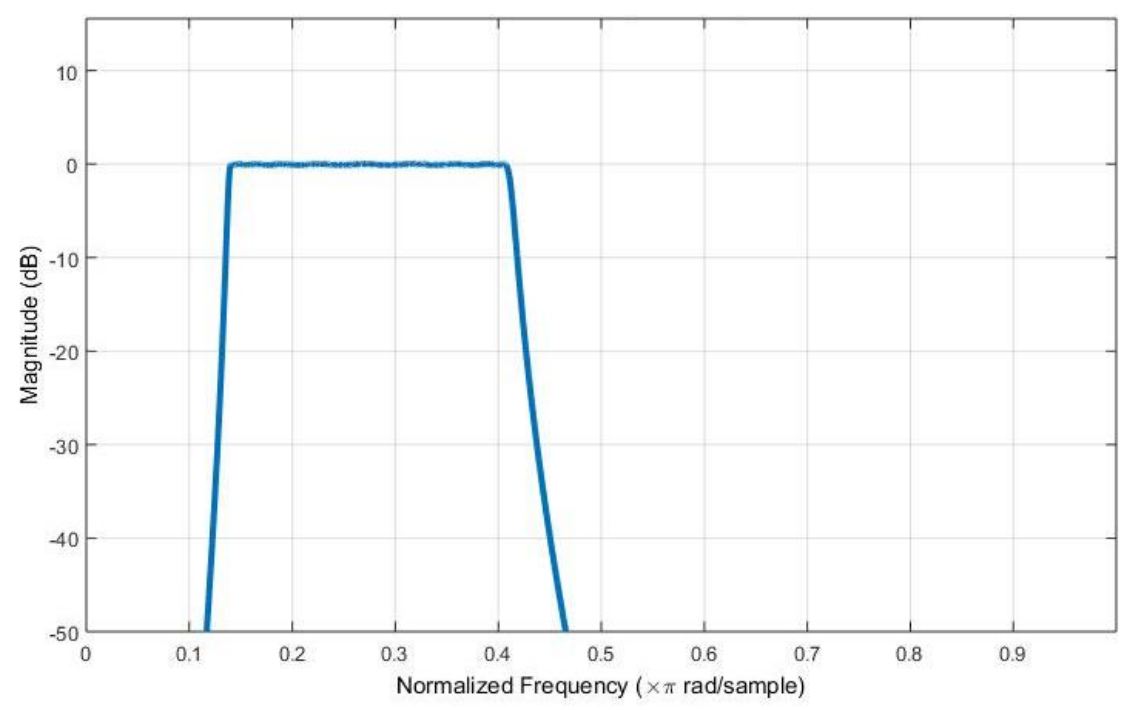

Figure 3.3: Magnitude response of a bandpass Chebyshev Type 1 filter.

\subsection{PREPROCESSING OF THE INPUT DATA}

The preprocessing of the available data is of great importance for the machine learning stage and usually can significantly affect the overall success or failure of the application of a given classification problem [3]. A transformation may be necessary to eliminate insignificant variations and superfluous details while at the same time accentuating the pertinent information [7].

Previous research where the moments of the LFM signals and BWGN were studied, suggests that the frequency-domain moments characterize a signal quite efficiently [24]. In our case we opt to use the spectra of the jamming signals, which are obtained by calculating the magnitude square of their Discrete Fourier Transform. Figure 3.4 and Figure 3.5 show the spectra of both a LFM signal and BWGN, respectively. The LFM signal is slightly corrupted with additive Gaussian white noise (30 dB SNR). Notice that the shapes of the spectra are quite distinct from each other. The Power Spectral Density (PSD) of a LFM signal exhibits a characteristic rectangular shape with high peaks on both ends of the bandwidth, while the PSD of BWGN has no discernible shape and high peaks occur anywhere inside the bandwidth. 


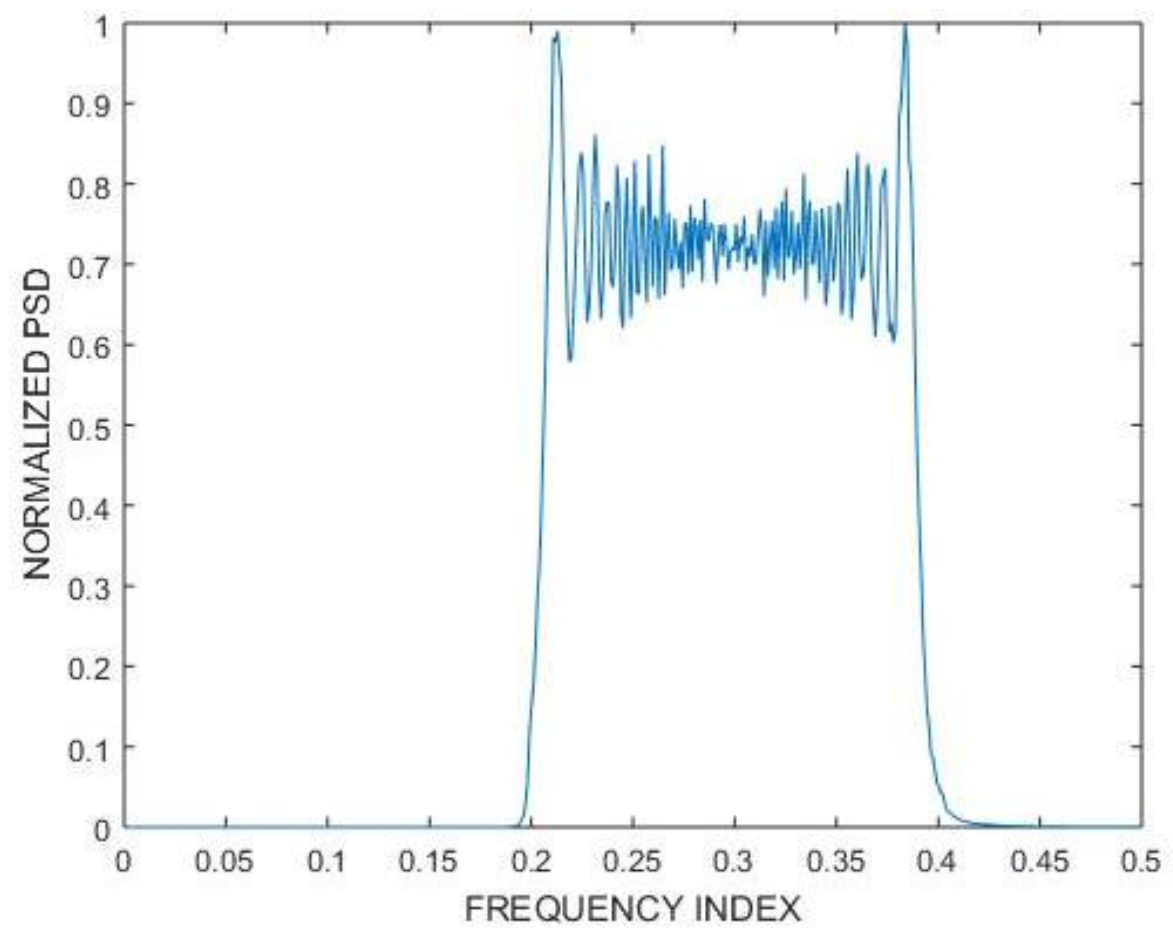

Figure 3.4: Power spectrum of a LFM signal with $\mathrm{SNR}=30 \mathrm{~dB}, \beta_{\mathrm{n}}=0.2$ and $\mathrm{f}_{\mathrm{n}}=0.2$.

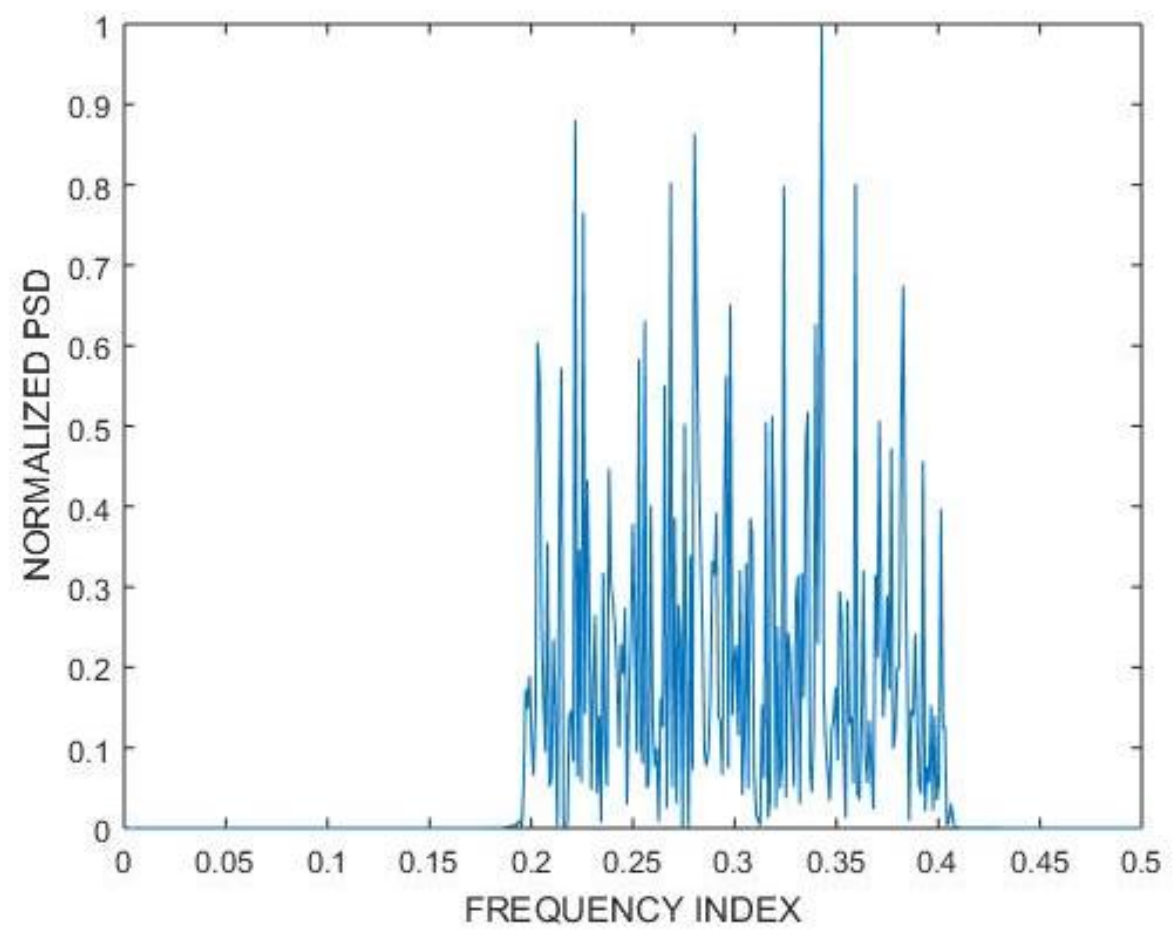

Figure 3.5: Power spectrum of BWGN with $\beta_{\mathrm{n}}=0.2$ and $\mathrm{f}_{\mathrm{n}}=0.2$. 
The preprocessing stage usually encompasses a feature extraction phase where the most distinctive characteristics of the input data are extracted and used as the input to the NN. In our work, this phase is omitted, for our purpose is to allow the NN to classify solely on the shape of the spectrum of the LFM signals and BWGN. Therefore, all the spectra are normalized to their corresponding highest peak to prevent the power amplitude of each spectrum from becoming another changing variable. In contrast to the previous LFM example, a $5 \mathrm{~dB}$ SNR is used for the LFM jamming signal shown in Fig. 3.6. Notice that the spectra in Figures 3.6 and 3.7, which exhibit the same normalized bandwidth and initial normalized frequency, start to lose their rectangular envelope, which can cause the NN to misclassify the signal.

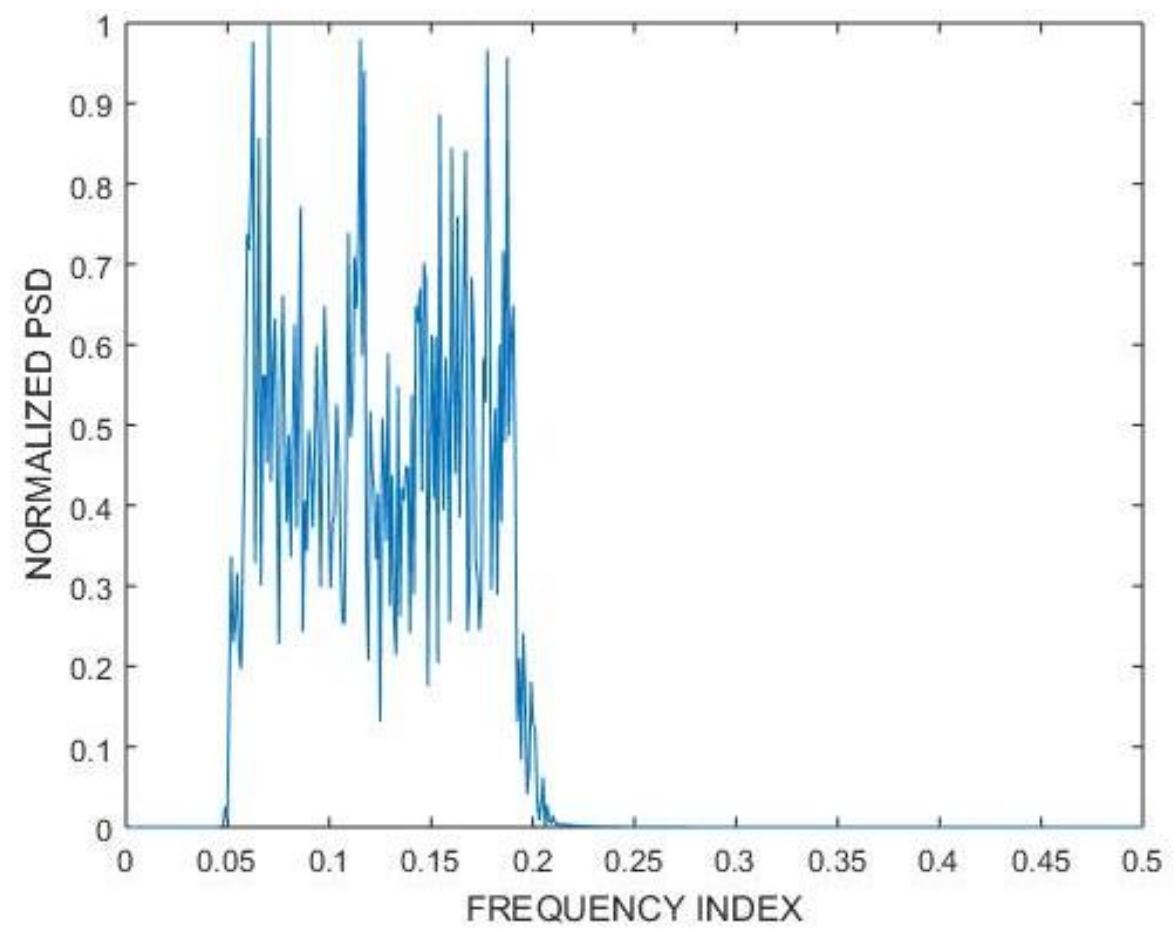

Figure 3.6: Power spectrum of a $L F M$ signal with $S N R=5 d B, \beta_{n}=0.15$, and $f_{n}=0.05$. 


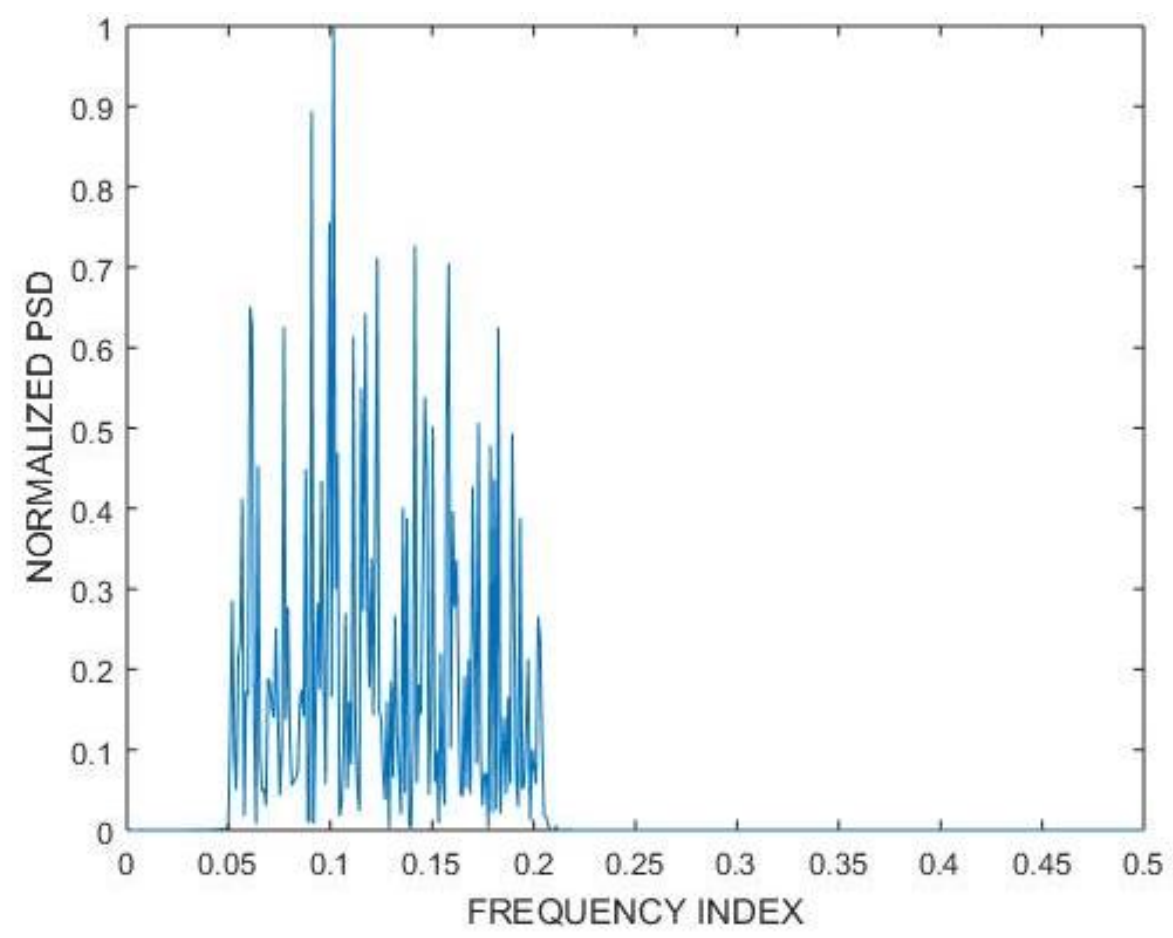

Figure 3.7: Power spectrum of BWGN with $\beta_{\mathrm{n}}=0.15$ and $\mathrm{f}_{\mathrm{n}}=0.05$.

\subsection{ORgANIZATION OF INPUT DATA INTO VECTOR FoRM}

To utilize the generated data we have to structure the information in an organized manner so that it can be easily input into the NN. As mentioned in section 2.4, the input data is organized in vector form and the elements of the vector dictate the size of the input layer. Accordingly, as illustrated in Figure 3.8, the generated spectra are then used to populate a matrix containing M spectra with $\mathrm{N}$ samples per spectra. Every column contained in this matrix is a placeholder for a single spectrum, and all the samples that make up each spectrum are stored within the row. The first half of the columns found in this matrix hold the spectra of LFM signals and the second half of the columns hold the spectra of the BWGN.

The matrix that holds the desired output values for each pattern (spectra in our case) that are input into the NN is known as the target matrix. Petrov et al. [3] show that utilizing two output 
neurons when classifying between two classes yields slightly better results than using one output neuron. Therefore, using two output neurons we encode the LFM jamming signals with the value of $(1,0)$ and BWGN with the value of $(0,1)$. The matrix shown in Figure 3.9 holds the NN output classification codes used to distinguish between a LFM signal and BWGN. Subsequently, the structure of our target matrix is made of two rows while the number of columns equals the number of columns in the input matrix. A value of 1 in the first row and a 0 in the second is used to classify a LFM jamming signal. A 0 in the first row and a 1 in the second is used to classify BWGN.

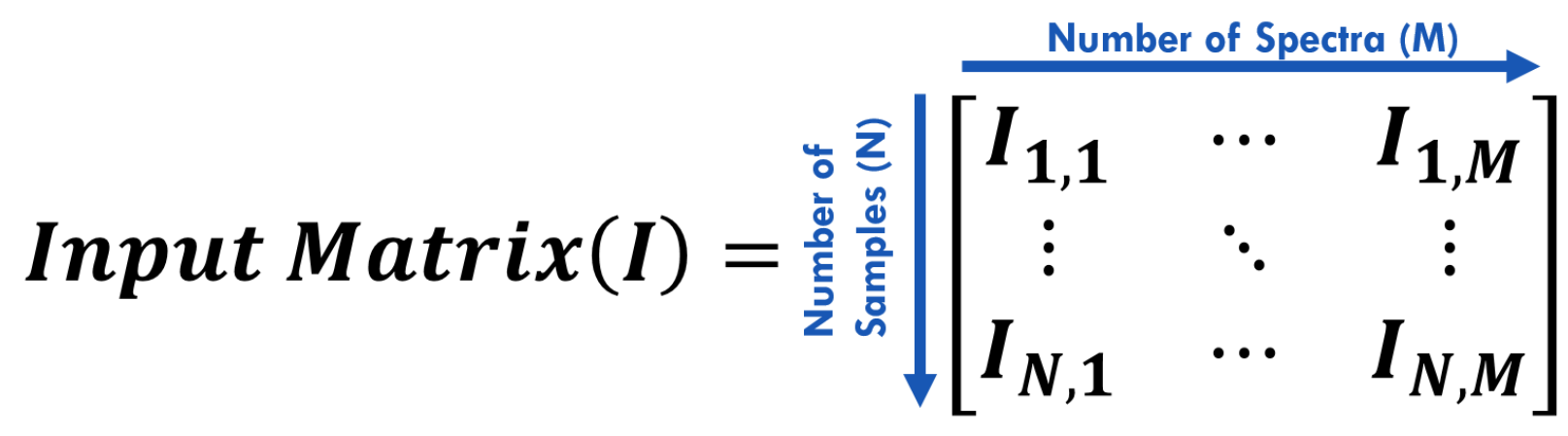

Figure 3.8: Organization of power spectra into a NxM input matrix.

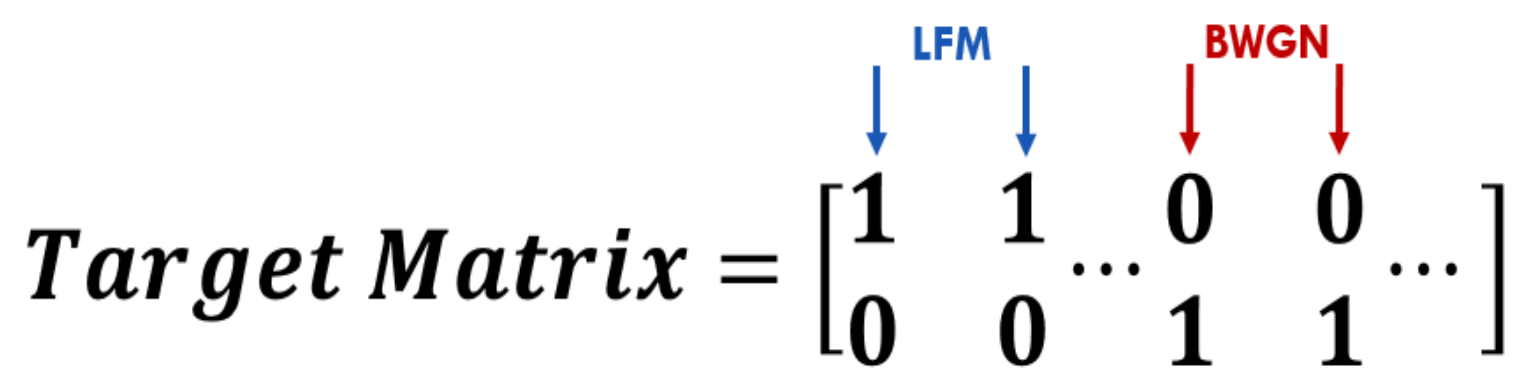

Figure 3.9: Target matrix containing the classification codes used to distinguish between LFM signals and $\mathrm{BWGN}(10=\mathrm{LFM}, 01=\mathrm{BWGN})$. 
The input matrix, along with its corresponding target matrix, is used as follows: $70 \%$ for training, $15 \%$ for validation and $15 \%$ for testing. The training set is used to adjust the internal weights of the NN when the input does not match the target answer, the validation set oversees the training process, and lastly, the testing set is used to assess the success of the NN classification capability. 


\section{Chapter 4: Analysis of Multiple Neural Network Architectures and Learning}

\section{Algorithms}

Thus far, to perform jamming signal classification, we have identified the multilayered feedforward network topology as our choice for neuron arrangement and interconnection. In this chapter, we conduct three experiments to examine the effects of varying NN architectures and learning algorithms for the application of classifying LFM jamming signals against BWGN. The first experiment deals with finding an optimal number of hidden neurons (Figure 4.1), the second experiment deals with finding an optimal number of hidden layers (Figure 4.2), and lastly, the third experiment deals with finding the most efficient learning algorithm.

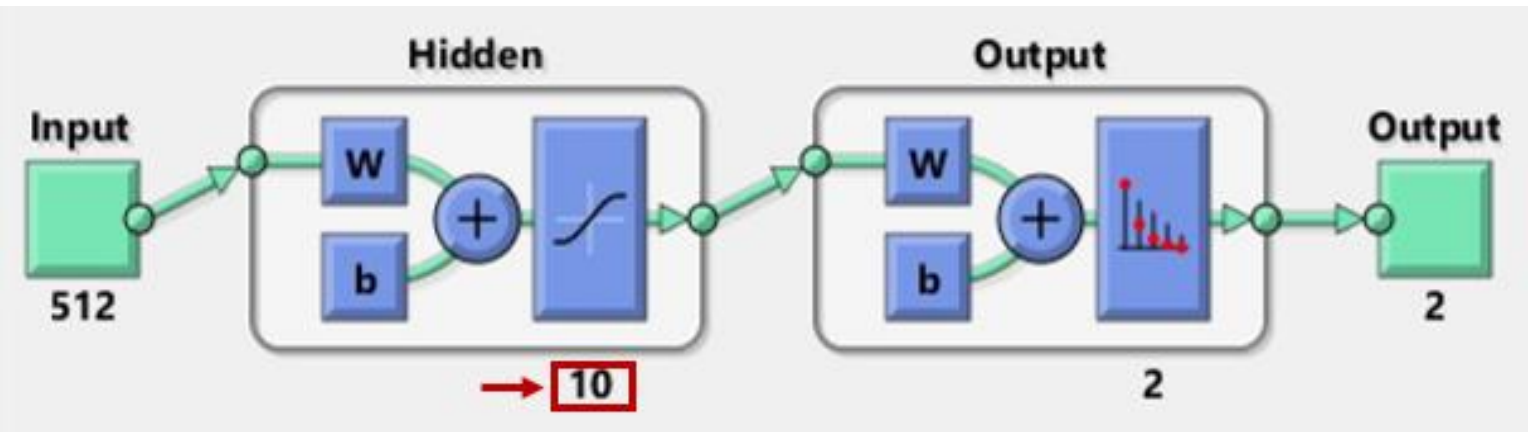

Figure 4.1: Neural network diagram with a focus on hidden neurons.

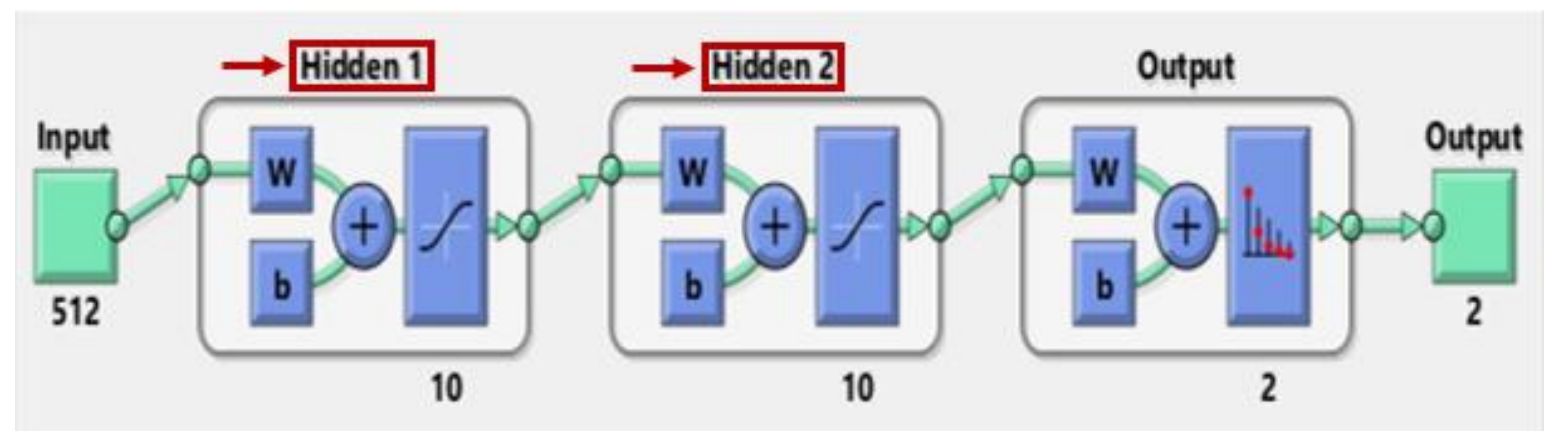

Figure 4.2: Neural network diagram with a focus on hidden layers. 
Before beginning this analysis, recall that a few fixed parameters have been defined and established in Chapter 2 for utilization in all experiments. To recap, due to its ability to accelerate convergence and reduce training time, the hyperbolic tangent sigmoid activation function is applied to all hidden neurons. Secondly, as it is favorable to heavily penalize outputs that are extremely inaccurate while slightly penalizing fairly correct classifications, a cross-entropy error function is used to evaluate the learning performance during weight adjustments. Lastly, we implement a supervised learning scenario for the training phase of all the simulations. Furthermore, the first two experiments utilize the SCG learning algorithm to carry out the training portion. The motive for this reasoning is discussed in section 4.1.

To preserve the generalization capability of the network and avoid overtraining, the criteria to halt the training phase is set to 1000 training epochs or 6 consequent validation check fails, whichever occurs first. In the first stopping condition, a training epoch denotes one forward pass and one backward pass of all the training data. To elaborate on the latter stopping condition, the NN uses the validation set (15\% of the input data) during training to assess how well the network is currently performing, if the network performance on the validation vectors remains the same or fails to improve for six consecutive number of epochs, the training will be halted.

Finally, the softmax activation function is used in all simulations for both neurons in the output layer. This activation function is used to represent a probability distribution across discrete mutually exclusive alternatives and is commonly used in conjunction with the cross-entropy error function.

The same input data matrix is used to train, validate, and test all the networks. Throughout the training phase, the network adjusts its weights depending on the patterns being inputted. If the network uses all the LFM signals to train during the beginning half of the training phase and all 
the BWGN for the second half, it runs the risk of forgetting all the weight adjustments that are calculated for the patterns of the previous LFM signals. For this reason, the input matrix is randomly divided using a split-sample technique to ensure a good generalization ability of the model. In addition, all input and target data are mapped/normalized from their original range to the range of -1 to 1 . The input matrix contains a total of 4096 spectra, each having a length of 512 samples and an SNR of $5 \mathrm{~dB}$. We found that for an SNR of at least $30 \mathrm{~dB}$, this number of spectra and samples yields a PD of 100\%, making it suitable for additional testing.

The success of the each experiment is measured in terms of the PD and PFA for the LFM signal. The PD is denoted as

$$
P_{D}=P(10 \text { output } \mid 10 \text { input })
$$

which is the probability of classifying a LFM signal when in fact it is a LFM signal. The PFA is denoted as

$$
P_{F A}=P(10 \text { output } \mid 01 \text { input })
$$

which is the probability of classifying a LFM signal when in fact it is not a LFM signal. Presented in Figure 4.3, a sample confusion matrix is shown for the network with an architecture of 512-102 (512 inputs, ten neurons in the hidden layer and two neurons in the output layer). The correct classification is seen in the green squares and the incorrect in the red squares. The upper number (green number) in the first square of the third row denotes the PD, the lower number (red number) in the second square of the third row denotes the PFA. 


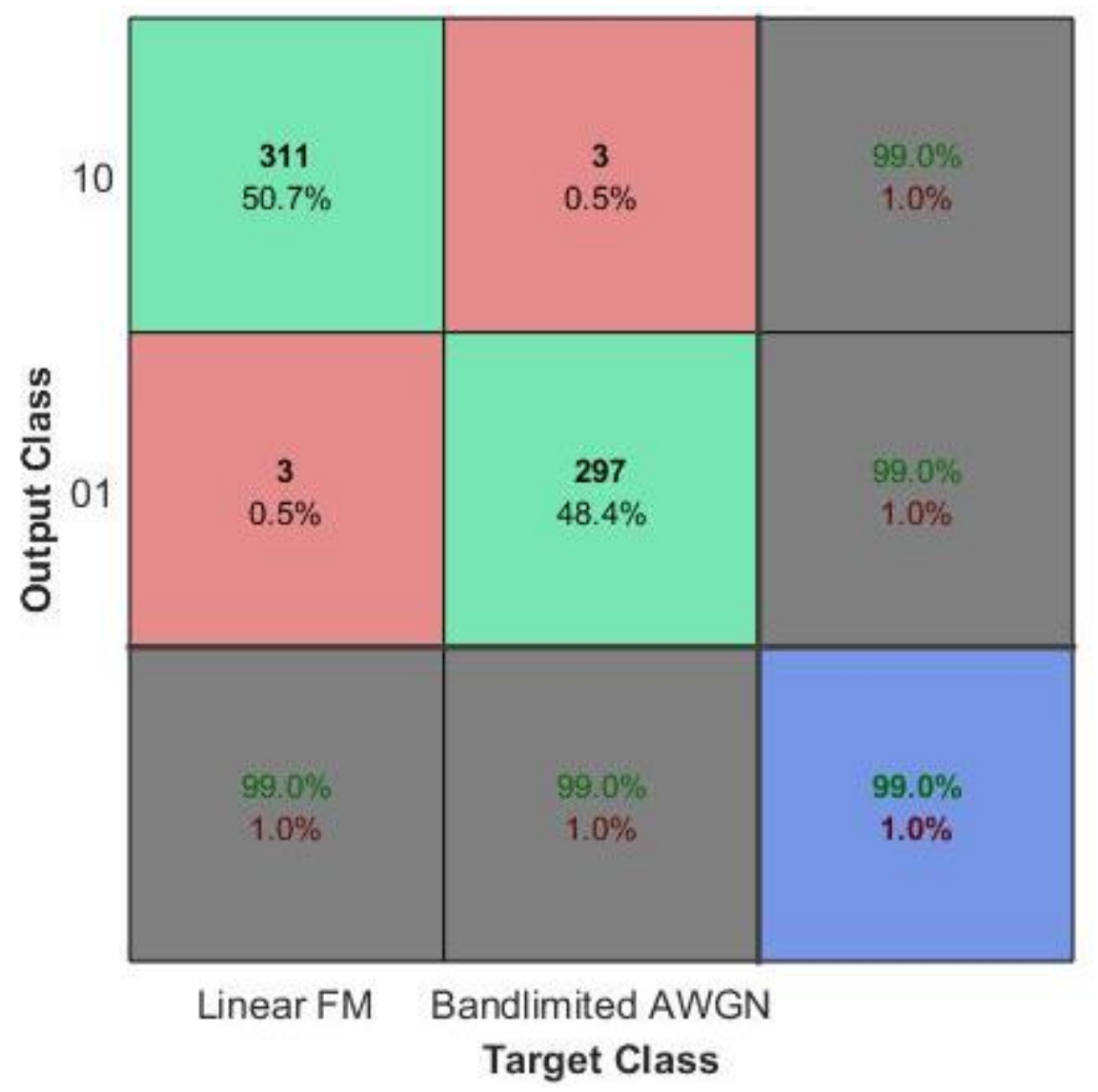

Figure 4.3: Sample confusion matrix for a neural network architecture of 512-10-2.

\subsection{Neural Network Architecture with Varying Number of Hidden NeUrons}

The first case study investigates the effects of altering the number of hidden neurons located in the hidden layer. For the rest of the architecture, the network is set up with one input layer, one hidden layer, and one output layer. The number of hidden neurons is varied from 1 to 40 , and each test is an average of 24 runs. Table 1 shows that an architecture containing any number between 1 and 5 hidden neurons yields a performance that does not meet our metrics $(95 \%<\mathrm{PD}$ and $1.5 \%>\mathrm{PFA})$. In particular, 1 or 2 hidden neurons are the worst architecture options, yielding a poor PD and a high PFA. When using an architecture ranging from 6 to 9 hidden neurons, the system begins to demonstrate an acceptable classification performance; if computational cost is of 
interest, utilizing these architectures will generate good results while the number of computations is kept to a minimum. Although results are deemed acceptable, the PD and PFA can vary in and out of our metric range. Any number above 10 neurons yields an average PD of $98.53 \pm 0.01 \%$ and an average PFA of $1.70 \% \pm 0.01$. The effect of increasing the number of neurons past the value of 10 is better appreciated in Figure 4.4, where the performance is shown to plateau and hold a constant average. The best performance is reached when using 32 neurons where the PD and PFA are $99.62 \% \pm 0.01$ and $1.07 \% \pm 0.01$, respectively. The case of 26 neurons deviates from the emerging pattern as the PFA spikes to $9.06 \%$. Generally speaking, however, having 10 or more hidden neurons yields good results.

Table 4.1: Neural network performance with varying number of hidden neurons.

\begin{tabular}{|c|c|c|c|c|}
\hline Neurons & PD & STD & PFA & STD \\
\hline 1 & $78.15 \%$ & 0.31 & $13.95 \%$ & 0.33 \\
\hline 2 & $84.12 \%$ & 0.20 & $6.10 \%$ & 0.20 \\
\hline 3 & $91.06 \%$ & 0.08 & $2.22 \%$ & 0.02 \\
\hline 4 & $95.07 \%$ & 0.06 & $1.63 \%$ & 0.01 \\
\hline 5 & $91.94 \%$ & 0.11 & $6.10 \%$ & 0.20 \\
\hline 6 & $96.74 \%$ & 0.05 & $1.26 \%$ & 0.01 \\
\hline 7 & $93.65 \%$ & 0.20 & $1.50 \%$ & 0.01 \\
\hline 8 & $97.93 \%$ & 0.04 & $1.62 \%$ & 0.01 \\
\hline 9 & $98.26 \%$ & 0.02 & $1.65 \%$ & 0.02 \\
\hline 10 & $97.41 \%$ & 0.03 & $1.29 \%$ & 0.01 \\
\hline 11 & $97.46 \%$ & 0.05 & $1.19 \%$ & 0.01 \\
\hline 12 & $98.62 \%$ & 0.02 & $1.33 \%$ & 0.01 \\
\hline 13 & $95.55 \%$ & 0.14 & $0.94 \%$ & 0.01 \\
\hline 14 & $99.20 \%$ & 0.01 & $1.03 \%$ & 0.01 \\
\hline 15 & $99.00 \%$ & 0.01 & $1.19 \%$ & 0.01 \\
\hline 16 & $98.92 \%$ & 0.01 & $1.33 \%$ & 0.01 \\
\hline 17 & $99.19 \%$ & 0.01 & $1.32 \%$ & 0.01 \\
\hline 18 & $99.01 \%$ & 0.02 & $1.53 \%$ & 0.02 \\
\hline 19 & $99.15 \%$ & 0.02 & $1.06 \%$ & 0.01 \\
\hline 20 & $99.31 \%$ & 0.01 & $1.15 \%$ & 0.02 \\
\hline & & & & \\
\hline
\end{tabular}

\begin{tabular}{|c|c|c|c|c|}
\hline Neurons & PD & STD & PFA & STD \\
\hline 21 & $98.80 \%$ & 0.03 & $1.58 \%$ & 0.01 \\
\hline 22 & $98.92 \%$ & 0.01 & $0.97 \%$ & 0.01 \\
\hline 23 & $99.48 \%$ & 0.01 & $1.63 \%$ & 0.01 \\
\hline 24 & $99.23 \%$ & 0.01 & $1.24 \%$ & 0.01 \\
\hline 25 & $99.53 \%$ & 0.01 & $1.12 \%$ & 0.01 \\
\hline 26 & $98.83 \%$ & 0.03 & $9.06 \%$ & 0.24 \\
\hline 27 & $96.40 \%$ & 0.13 & $3.98 \%$ & 0.14 \\
\hline 28 & $99.41 \%$ & 0.01 & $1.21 \%$ & 0.01 \\
\hline 29 & $98.98 \%$ & 0.01 & $1.44 \%$ & 0.02 \\
\hline 30 & $99.40 \%$ & 0.01 & $1.10 \%$ & 0.01 \\
\hline 31 & $98.30 \%$ & 0.02 & $1.41 \%$ & 0.01 \\
\hline 32 & $99.62 \%$ & 0.01 & $1.07 \%$ & 0.01 \\
\hline 33 & $99.24 \%$ & 0.01 & $1.19 \%$ & 0.01 \\
\hline 34 & $98.94 \%$ & 0.01 & $1.75 \%$ & 0.02 \\
\hline 35 & $95.10 \%$ & 0.01 & $1.42 \%$ & 0.01 \\
\hline 36 & $98.43 \%$ & 0.04 & $1.13 \%$ & 0.01 \\
\hline 37 & $95.99 \%$ & 0.16 & $2.39 \%$ & 0.05 \\
\hline 38 & $98.18 \%$ & 0.06 & $3.05 \%$ & 0.07 \\
\hline 39 & $99.33 \%$ & 0.01 & $1.20 \%$ & 0.01 \\
\hline 40 & $99.40 \%$ & 0.01 & $1.26 \%$ & 0.01 \\
\hline & & & & \\
\hline
\end{tabular}




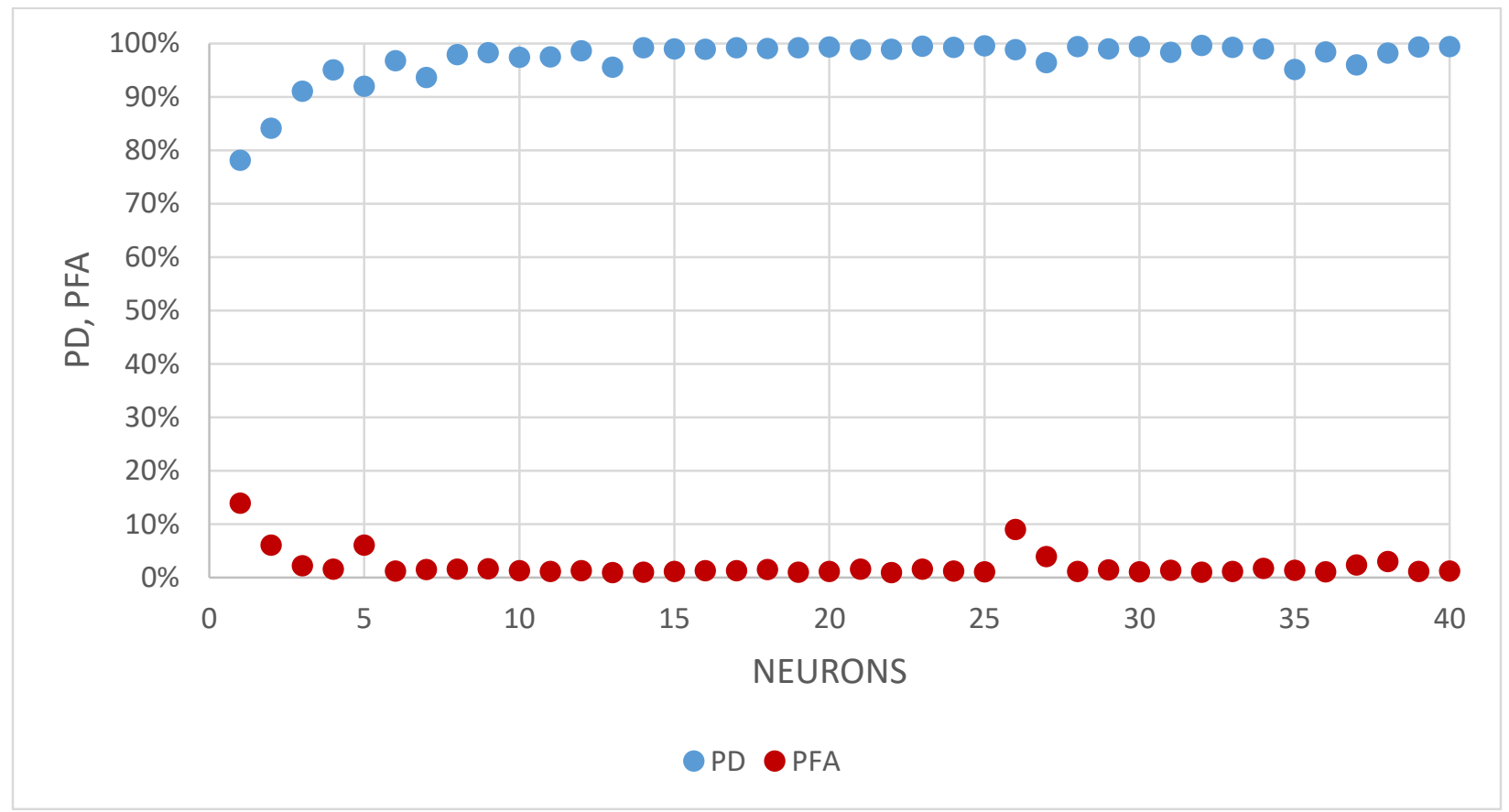

Figure 4.4: Plot of Table 4.1.

Artificial NNs cannot function without a learning algorithm, therefore one must be selected to perform the test that will determine the most efficient number of hidden neurons and hidden layers. We previously mentioned that the SCG learning algorithm is our preferred choice. To support our decision, four additional experiments are carried out. Each of the other learning algorithms is tested with $1,3,5,7,9$, and 11 hidden neurons to see if any can outperform the SCG learning algorithm. Since each architecture performance is averaged over 24 runs, Tables 4.2-4.5 show the standard deviations of the PD and PFA measurements for each of these experiments. Notice that when using 11 hidden neurons, these algorithms do not yield a PD of 95\% or higher and a PFA of $1.5 \%$ or lower. Additional comparisons are discussed in section 4.3 where the different learning algorithms are examined while having the same neural architecture. 
Table 4.2: Neural network performance for the Resilient Backpropagation learning algorithm with varying number of hidden neurons.

\begin{tabular}{|c|c|c|c|c|}
\hline Neurons & PD & STD & PFA & STD \\
\hline 1 & $60.58 \%$ & 0.42 & $44.63 \%$ & 0.47 \\
\hline 3 & $50.35 \%$ & 0.39 & $19.75 \%$ & 0.37 \\
\hline 5 & $67.98 \%$ & 0.37 & $34.91 \%$ & 0.45 \\
\hline 7 & $61.88 \%$ & 0.37 & $15.38 \%$ & 0.33 \\
\hline 9 & $60.85 \%$ & 0.38 & $26.53 \%$ & 0.42 \\
\hline 11 & $65.90 \%$ & 0.40 & $31.13 \%$ & 0.44 \\
\hline
\end{tabular}

Table 4.3: Neural network performance for the Conjugate Gradient with Powell/Beale Restarts learning algorithm with varying number of hidden neurons.

\begin{tabular}{|c|c|c|c|c|}
\hline Neurons & PD & STD & PFA & STD \\
\hline 1 & $56.03 \%$ & 0.44 & $17.74 \%$ & 0.37 \\
\hline 3 & $78.15 \%$ & 0.28 & $10.64 \%$ & 0.27 \\
\hline 5 & $87.70 \%$ & 0.12 & $6.41 \%$ & 0.20 \\
\hline 7 & $93.16 \%$ & 0.16 & $1.70 \%$ & 0.02 \\
\hline 9 & $91.95 \%$ & 0.10 & $1.68 \%$ & 0.01 \\
\hline 11 & $93.10 \%$ & 0.12 & $2.02 \%$ & 0.02 \\
\hline
\end{tabular}

Table 4.4: Neural network performance for the Polak-Ribiére Conjugate Gradient learning algorithm with varying number of hidden neurons.

\begin{tabular}{|c|c|c|c|c|}
\hline Neurons & PD & STD & PFA & STD \\
\hline 1 & $70.79 \%$ & 0.41 & $34.30 \%$ & 0.46 \\
\hline 3 & $82.39 \%$ & 0.19 & $11.08 \%$ & 0.27 \\
\hline 5 & $86.23 \%$ & 0.22 & $12.83 \%$ & 0.30 \\
\hline 7 & $92.81 \%$ & 0.09 & $5.45 \%$ & 0.19 \\
\hline 9 & $95.40 \%$ & 0.05 & $1.53 \%$ & 0.01 \\
\hline 11 & $95.04 \%$ & 0.08 & $1.59 \%$ & 0.02 \\
\hline
\end{tabular}


Table 4.5: Neural network performance for the Variable Learning Rate Backpropagation learning algorithm with varying number of hidden neurons.

\begin{tabular}{|c|c|c|c|c|}
\hline Neurons & PD & STD & PFA & STD \\
\hline 1 & $63.63 \%$ & 0.38 & $5.29 \%$ & 0.20 \\
\hline 3 & $91.05 \%$ & 0.07 & $2.70 \%$ & 0.06 \\
\hline 5 & $90.28 \%$ & 0.13 & $4.94 \%$ & 0.15 \\
\hline 7 & $95.14 \%$ & 0.06 & $2.44 \%$ & 0.07 \\
\hline 9 & $93.53 \%$ & 0.15 & $1.30 \%$ & 0.02 \\
\hline 11 & $94.01 \%$ & 0.11 & $1.46 \%$ & 0.03 \\
\hline
\end{tabular}

\subsection{NeUral Network ArChitecture With VARYing Number OF Hidden LAYERS}

The second case study investigates the effects of altering the number of hidden layers inside a NN. Five cases are examined where the NN contains 1, 2, 3, 4, and 5 hidden layers in each instance. As for the rest of the architecture, the network is set up with one input layer and one output layer. The computational burden increases as the number of hidden layers and hidden neurons increase, for this reason, the number of hidden neurons is set to 10 in each hidden layer to promptly advance the training phase. Since optimal results of PD and PFA are reached with 10 hidden neurons, it is decided to proceed the experiment with that value. As in the previous experiment, every result is an average of 24 runs.

Furthermore, it can be observed from Figure 4.5 that changing the number of hidden layers does not have an impact on the classification; with the exception of the architecture with 4 hidden layers, all the cases achieved a PD higher than 97\%. Table 4.6 shows the standard deviations of the PD and PFA measurements for each architecture. The architecture containing 1 hidden layer appears to have the highest PD with $99.35 \% \pm 0.00$ and the lowest PFA with $0.78 \% \pm 0.01$. The only downfall of utilizing more hidden layers is that the time spent training the NN increases due to the additional calculations needed for the extra layers. If training time is an issue, one should 
consider using less hidden layers. Notice that using 4 hidden layers yields statistically significant inferior results.

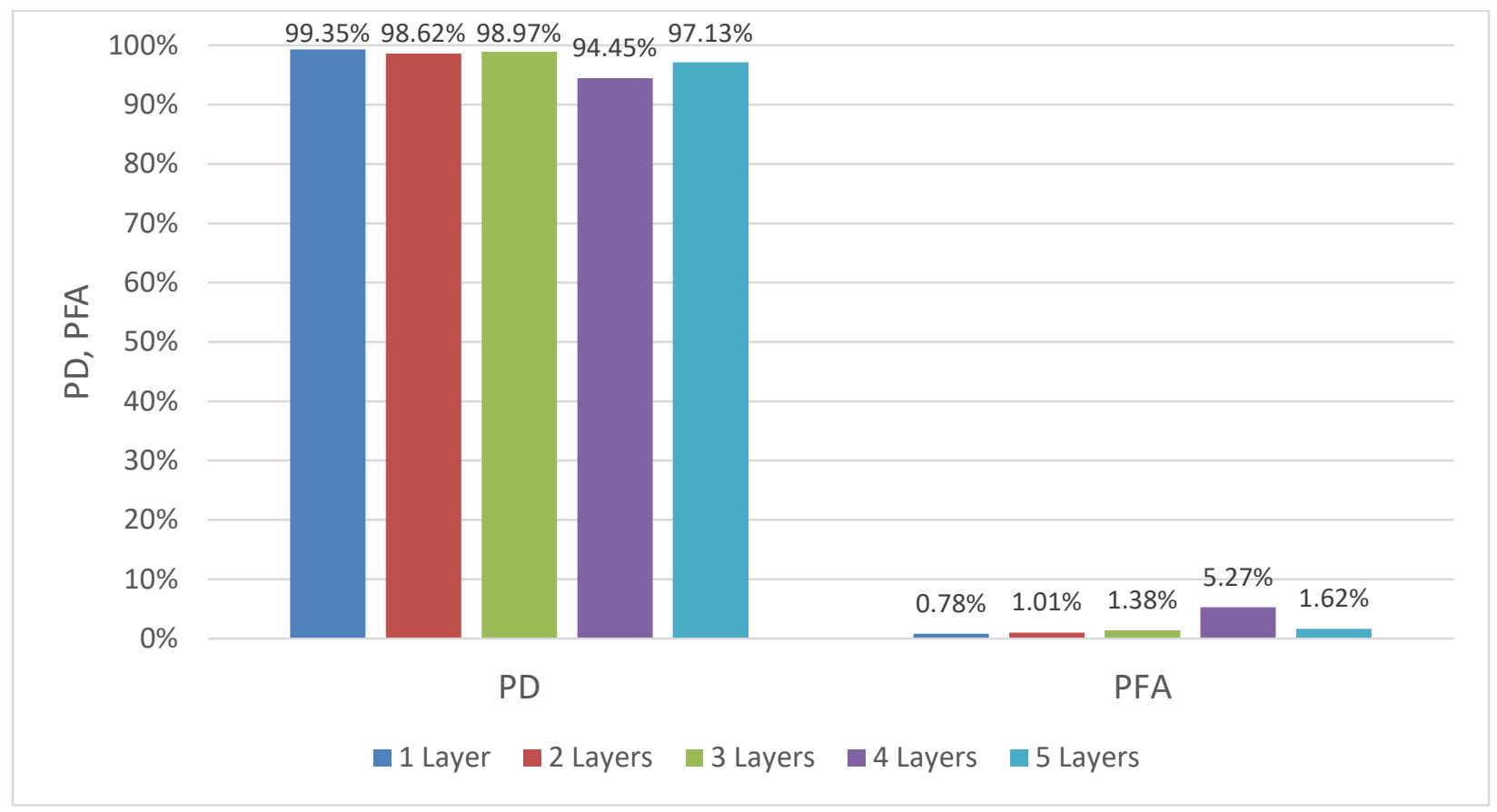

Figure 4.5: Neural network performance with varying number of hidden layers.

Table 4.6: Standard deviations of PD and PFA measurements for different layers.

\begin{tabular}{|c|c|c|c|c|}
\hline Layers & PD & STD & PFA & STD \\
\hline 1 & $99.35 \%$ & 0.00 & $0.78 \%$ & 0.01 \\
\hline 2 & $98.62 \%$ & 0.01 & $1.01 \%$ & 0.01 \\
\hline 3 & $98.97 \%$ & 0.01 & $1.38 \%$ & 0.01 \\
\hline 4 & $94.45 \%$ & 0.20 & $5.27 \%$ & 0.20 \\
\hline 5 & $97.13 \%$ & 0.06 & $1.62 \%$ & 0.01 \\
\hline
\end{tabular}

\subsection{Assessment of Different Learning Algorithms for a Multilayer Network}

The final case study investigates the effects of changing the learning algorithm utilized to adjust the weights within a NN. Recall that the learning algorithms explored in this research are: 
Resilient Backpropagation (RP), Scale Conjugate Gradient (SCG), Conjugate Gradient with Powell/Beale Restarts (CGB), Polak-Ribiére Conjugate Gradient (CGP), and the Variable Learning Rate Backpropagation (GDX). Training of the NN occurs according to the training parameters of each learning algorithm which are shown in Tables 4.7-4.11 with their default values. Keep in mind that some algorithms make use of different parameters to train the network.

Table 4.7: Training parameters for the Resilient Backpropagation (RP) learning algorithm.

\begin{tabular}{|l|l|}
\hline 1000 & Maximum number of epochs to train \\
\hline 0 & Performance goal \\
\hline infinity & Maximum time to train in seconds \\
\hline 6 & Maximum validation failures \\
\hline $1 \mathrm{E}-05$ & Minimum performance gradient \\
\hline 0.01 & Learning rate \\
\hline 1.2 & Increment to weight change (delta_inc) \\
\hline 0.5 & Decrement to weight change (delta_dec) \\
\hline 0.07 & Initial weight change (delta) \\
\hline 50 & Maximum weight change (delta_max) \\
\hline
\end{tabular}

Table 4.8: Training parameters for the Scaled Conjugate Gradient (SCG) learning algorithm.

\begin{tabular}{|l|l|}
\hline 1000 & Maximum number of epochs to train \\
\hline 0 & Performance goal \\
\hline infinity & Maximum time to train in seconds \\
\hline 6 & Maximum validation failures \\
\hline $1 \mathrm{E}-06$ & Minimum performance gradient \\
\hline 0.00005 & $\begin{array}{l}\text { Determine change in weight for second derivative approximation } \\
\text { (sigma) }\end{array}$ \\
\hline $5 \mathrm{E}-07$ & Parameter for regulating the indefiniteness of the Hessian (lambda) \\
\hline
\end{tabular}


Table 4.9: Training parameters for the Conjugate Gradient with Powell/Beale Restarts (CGB) learning algorithm.

\begin{tabular}{|l|l|}
\hline 1000 & Maximum number of epochs to train \\
\hline 0 & Performance goal \\
\hline infinity & Maximum time to train in seconds \\
\hline 6 & Maximum validation failures \\
\hline $1 \mathrm{E}-10$ & Minimum performance gradient \\
\hline
\end{tabular}

Table 4.10: Training parameters for the Variable Learning Rate Backpropagation (GDX) learning algorithm.

\begin{tabular}{|l|l|}
\hline 1000 & Maximum number of epochs to train \\
\hline 0 & Performance goal \\
\hline infinity & Maximum time to train in seconds \\
\hline 6 & Maximum validation failures \\
\hline 0.01 & Learning rate \\
\hline 1.05 & Ratio to increase learning rate \\
\hline 0.7 & Ratio to decrease learning rate \\
\hline 1.04 & Maximum performance increase \\
\hline 0.9 & Momentum constant \\
\hline $1 \mathrm{E}-05$ & Minimum performance gradient \\
\hline
\end{tabular}

Table 4.11: Training parameters for the Polak-Ribiére Conjugate Gradient (CGP) learning algorithm.

\begin{tabular}{|l|l|}
\hline 1000 & Maximum number of epochs to train \\
\hline 0 & Performance goal \\
\hline infinity & Maximum time to train in seconds \\
\hline 6 & Maximum validation failures \\
\hline $1 \mathrm{E}-10$ & Minimum performance gradient \\
\hline
\end{tabular}


Unlike the previous two experiments, there is a clear distinction of performance when varying between these five algorithms. A summary of the obtained results is presented in Figure 4.6. Similarly, every learning algorithm performance is an average of 24 runs. Table 4.12 shows the standard deviations of the PD and PFA measurements for each learning algorithm. The highest performing algorithm is clearly the Scaled Conjugate Gradient, achieving the highest PD with a value of $99.35 \% \pm 0.00$ and also the lowest PFA with a value of $0.78 \% \pm 0.01$. The RP displayed the lowest performance with a PD and a PFA of $62.93 \% \pm 0.40$ and $14.14 \% \pm 0.32$, respectively. Though, the CGB algorithm did not perform as poorly it is not an optimal learning algorithm for our application since the PD was lower than 95\%. For optimality, choosing the SCG, CGP or the GDX learning algorithms, will suffice.

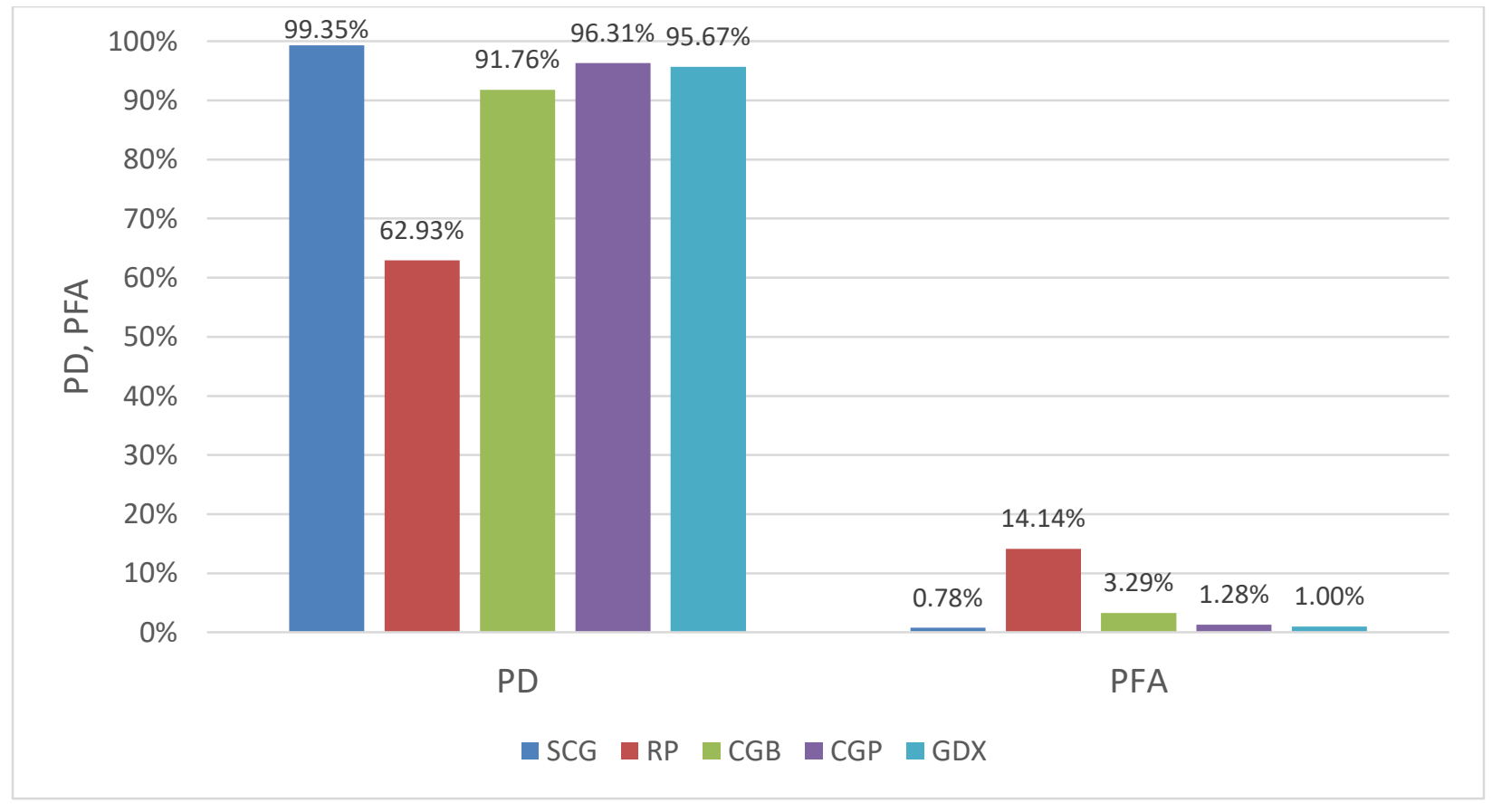

Figure 4.6: Assessment of neural network performance with different learning algorithms. 
Table 4.12: Standard deviations of PD and PFA measurements for different learning algorithms.

\begin{tabular}{|c|c|c|c|c|}
\hline Algorithm & PD & STD & PFA & STD \\
\hline SCG & $99.35 \%$ & 0.00 & $0.78 \%$ & 0.01 \\
\hline RP & $62.93 \%$ & 0.40 & $14.14 \%$ & 0.32 \\
\hline CGB & $91.76 \%$ & 0.19 & $3.29 \%$ & 0.09 \\
\hline CGP & $96.31 \%$ & 0.05 & $1.28 \%$ & 0.01 \\
\hline GDX & $95.67 \%$ & 0.11 & $1.00 \%$ & 0.01 \\
\hline
\end{tabular}




\section{Chapter 5: Conclusions}

In this research, we chose a multilayer feedforward neural network as an alternative method for classifying LFM signals against BWGN and proposed a systematic approach to determine an optimal NN architecture capable of yielding a PD of $95 \%$ or higher and a PFA of $1.5 \%$ or lower at a $5 \mathrm{~dB}$ SNR.

A technique for generating a database of LFM signals was developed while the length for each jamming signal was kept at 512 samples. In doing so, we were able to control the normalized initial frequency and normalized bandwidth, which lie within the Nyquist criterion, for each LFM jamming signal. A Chebyshev Type 1 bandpass filter whose cutoff frequencies matched the normalized initial and final frequencies of the LFM signals was used to remove additive noise outside the signal bandwidth. Similarly, the same bandpass filter design was employed to generate BWGN from white noise.

The LFM signals and the BWGN were then processed into spectra. The spectra were later normalized to their highest peaks so that the absolute power was not considered in the classification scheme. Utilizing the shape of the spectra proved to be a suitable preprocessing method. Subsequently, a matrix was constructed with filtered and normalized spectra to easily feed the data into the NN.

Different architectures of neural networks with different learning algorithms were explored. Through simulation, the individual effects of changing the number of hidden neurons, the number of hidden layers, and the training algorithms were studied. We determined that an architecture with 10 hidden neurons (or higher), one hidden layer, and a Scaled Conjugate Gradient for training algorithm encapsulates an optimal architecture for our application. We verified that too many hidden neurons, say 25 or higher, eventually starts to produce unpredictable results in 
some instances where the system might oscillate between a good and bad performance. Nevertheless, this data aids the notion that having at least three times more hidden neurons than there are output neurons increases neural network performance [7]. Also, changing the number of hidden layers had no significant effect on the PD and PFA. Though, in the experiment that examined 4 hidden layers, the PFA did yield a surprising value of 5.27\%. Regardless, having 1 hidden layer did yield the highest PD with the lowest PFA and was deemed optimal due to its lower training time. Additionally, given that the Polak-Ribiére Conjugate Gradient and the Variable Learning Rate Backpropagation learning algorithms did yield a PD slightly higher than 95\% and a PFA lower than $1.5 \%$, the Scaled Conjugate Gradient algorithm surpassed the rest of the algorithms by nearly reaching a PD of $100 \%$.

It is difficult and very time consuming to design a NN, let alone design a network architecture that successfully works with different applications. To elaborate, a NN architecture that works for a signal classification problem might not be optimal for a target recognition problem. Often, there are too many parameters to consider; changing any parameter can, in some instances, greatly affect the performance of a NN while in others the effects can be deemed insignificant. It is worth remembering that the selection of the input data and how it is preprocessed can also greatly affect the performance of a NN. This work strived to ease the process of design by creating a theoretical framework where the user, with careful consideration, can determine which parameters are best left fixed and which can be modified.

Given the success of this study, more experimentation is recommended and additional architectural parameters need to be explored to create a functioning expert system capable of classifying jamming signals to aid in the effort of countermeasures. Future work should include the study of changing the activation functions within the hidden neurons, changing the number of 
validation checks fails for the stopping condition, and testing more training algorithms. We plan to extend the work by implementing additional signals like the power-law FM and the chaotic signal to greater challenge the $\mathrm{NN}$; by classifying between three or four classes we can better study the effects of changing the neural network's architecture. 


\section{References}

[1] M. Dubin, How the brain works, 1st ed. Malden, Mass.: Blackwell Science, 2002, pp. 35 .

[2] H. Demuth and M. Beale, Neural Network Toolbox ${ }^{\text {TM: }}$ Users's Guide (Version 4) [Online]. Available: http://www.image.ece.ntua.gr/courses_static/nn/matlab/nnet.pdf. [Accessed November 20 2016].

[3] Nedyalko Petrov, Ivan Jordanov, Jon Roe, Radar Emitter Signals Recognition and Classification with Feedforward Networks, Procedia Computer Science, Volume 22, 2013, Pages 1192-1200, ISSN 1877-0509.

[4] Marzban, C. and G.J. Stumpf, "A Neural Network for Tornado Prediction Based on Doppler Radar-Derived Attributes,” J. Appl. Meteor., 35, 617-626,1996.

[5] P. Jarabo-Amores, D. Mata-Moya, M. Rosa Zurera, J. C. Nieto-Borge and F. LópezFerreras, "A neural network approach to improve radar detector robustness," 2006 14th European Signal Processing Conference, Florence, 2006, pp. 1-5, 2006.

[6] K. Cheikh and F. Soltani, "Application of neural networks to radar signal detection in Kdistributed clutter," in IEE Proceedings - Radar, Sonar and Navigation, vol. 153, no. 5, pp. 460-466, Oct. 2006.

[7] C. Carter and N. Masse, "Neural Networks for Classification of Radar Signals", Defence Research Establishment Ottawa, pp. 19-35, 1993.

[8] S. C. Ahalt, F. D. Garber, I. Jouny, and A. K. Krishnamurthy. 1989. Performance of synthetic neural network classification of noisy radar signals. In Advances in neural information processing systems 1, David S. Touretzky (Ed.). Morgan Kaufmann Publishers Inc., San Francisco, CA, USA 281-288.

[9] I. Jordanov, N. Petrov and A. Petrozziello, "Supervised radar signal classification," 2016 International Joint Conference on Neural Networks (IJCNN), Vancouver, BC, 2016, pp. 1464-1471.

[10] B. M. Wilamowski, "Neural network architectures and learning algorithms," in IEEE Industrial Electronics Magazine, vol. 3, no. 4, pp. 56-63, Dec. 2009.

[11] L. K. Hansen and P. Salamon, "Neural network ensembles," in IEEE Transactions on Pattern Analysis and Machine Intelligence, vol. 12, no. 10, pp. 993-1001, Oct 1990.

[12] I. Kanellopoulos and G. G. Wilkinson, "Strategies and best practice for neural network image classification," International Journal of Remote Sensing, vol.18, no. 4, pp. 711$725,1997$.

[13] Y. Hara, R. G. Atkins, S. H. Yueh, R. T. Shin and J. A. Kong, "Application of neural networks to radar image classification," in IEEE Transactions on Geoscience and Remote Sensing, vol. 32, no. 1, pp. 100-109, Jan 1994.

[14] J. Dayhoff, Neural network architectures, 1st ed. New York: Van Nostrand Reinhold, 1990. 
[15] L. Fausett, Fundamentals of neural networks, 1st ed. Delhi, India: Pearson Education, 2004.

[16] MathWorks, Multilayer Neural Network Architecture - MATLAB \& Simulink [Online]. Available: https://www.mathworks.com/help/nnet/ug/multilayer-neural-networkarchitecture.html. [Accessed: 12- Dec- 2016].

[17] MathWorks, Hyperbolic tangent sigmoid transfer function - MATLAB tansig [Online]. Available: https://www.mathworks.com/help/nnet/ref/tansig.html. [Accessed: 13- Dec2016].

[18] M. H. Beale, H. B. Demuth and M. T. Hagan, Mathworks®, (2016). Neural Network

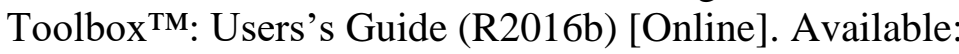
https://www.mathworks.com/help/pdf_doc/nnet/nnet_ug.pdf. [Accessed December 12, 2016]

[19] MathWorks, Mean squared normalized error performance function - MATLAB mse [Online]. Available: https://www.mathworks.com/help/nnet/ref/mse.html. [Accessed: 06Mar- 2017].

[20] MathWorks, Neural network performance - MATLAB crossentropy - MathWorks [Online]. Available: https://www.mathworks.com/help/nnet/ref/crossentropy.html\#namevaluepairs. [Accessed: 06- Mar- 2017].

[21] Y. Huang, P. V. Brennan and A. Seeds, "Active RFID location system based on timedifference measurement using a linear FM chirp tag signal," 2008 IEEE $19^{\text {th }}$ International Symposium on Personal, Indoor and Mobile Radio Communications, Cannes, 2008, pp. 1-5.

[22] M. Soumekh, "SAR-ECCM using phase-perturbed LFM chirp signals and DRFM repeat jammer penalization," in IEEE Transactions on Aerospace and Electronic Systems, vol. 42, no. 1, pp. 191-205, Jan. 2006.

[23] D. Salminen, "Adaptive filters applied on radar signals," Dissertation, 2013.

[24] A. Mendoza, "Classification of radar jammer FM signals using a neural network approach," Thesis, 2017. 


\section{Vita}

Alberto Soto was born in El Paso, Texas. He graduated summa cum laude with a Bachelor of Science in Electrical Engineering from the University of Texas at E1 Paso (UTEP) in 2016. That same year, Alberto entered the graduate school at UTEP seeking a Master of Science degree in Electrical Engineering. He worked on a project funded by the U.S. Army Research Laboratory. The project involved conducting research on artificial neural networks for the application of radar jamming signal classification under the supervision of Dr. Benjamin C. Flores. Alberto published and presented his research results at the Radar Sensor Technology exhibition of the SPIE Defense and Commercial Sensing conference in 2017. He is a student member of IEEE and SPIE as well as a member of the IEEE honor society Eta Kappa Nu (IEEE-HKN).

Contact Information: asoto21@miners.utep.edu

This thesis was typed by Alberto Soto. 